CAHIERS DE

NARRATOLOGIE

\section{Cahiers de Narratologie}

Analyse et théorie narratives

29 | 2015

Street Art 1

\title{
Les murs parlent de nous. Esthétique politique des singularités quelconques
}

Alain Bertho

\section{(2) OpenEdition}

\section{Journals}

\section{Electronic version}

URL: http://journals.openedition.org/narratologie/7410

DOI: 10.4000/narratologie.7410

ISSN: $1765-307 X$

\section{Publisher}

LIRCES

\section{Electronic reference}

Alain Bertho, «Les murs parlent de nous. Esthétique politique des singularités quelconques », Cahiers de Narratologie [Online], 29 | 2015, Online since 13 January 2016, connection on 19 April 2019. URL : http://journals.openedition.org/narratologie/7410; DOI : 10.4000/narratologie.7410

This text was automatically generated on 19 April 2019

Article L.111-1 du Code de la propriété intellectuelle. 


\title{
Les murs parlent de nous. Esthétique politique des singularités quelconques
}

\author{
Alain Bertho
}

1 Qui enquête sur les mobilisations urbaines contemporaines ne peut ignorer l'omniprésence des images: images partagées en ligne des voitures qui brûlent comme des affrontements des places de Tahrir à Taksim, images murales intempestives et parfois énigmatiques. Dans les deux cas l'héritage de l'anthropologie visuelle comme d'autres disciplines, qu'il s'agisse de l'infocom ou de l'histoire de l'art, ne nous est pas d'un grand secours pour décrypter ce nouvel usage pictural. Nous en sommes plus à l'inventaire de nos ignorances et de nos perplexités qu'à la convocation de nos savoirs constitués et à leurs catégories pour rendre compte de cette contemporanéité.

2 Concernant les graffiti et le Street art, il ne s'agira donc pas ici de répondre aux questions concernant leur nouveauté ou leur qualité artistique, ni leur mobilisation dans le marché de l'art ou les politiques culturelles urbaines, mais de comprendre (un peu) ce qu'il y a de contemporain dans ces pratiques et ce qu'elles nous disent de nous, des villes et de ceux qui y vivent.

3 L'ethnologie du contemporain s'adosse à l'hypothèse que la période que nous vivons est nouvelle et que la modernité des XIXème et XXème siècles est close. Il n'est pas ici le lieu d'argumenter cette hypothèse largement exposée dans d'autres publications ${ }^{1}$. Il s'agit de partir d'elle et de ses conséquences : les catégories et les paradigmes modernes sont peu opérants pour la compréhension du présent et c'est par l'enquête sur l'intellectualité du monde et même de son l'intellectualité populaire que nous pouvons en constituer de nouveaux.

4 Pour le sujet qui nous occupe, je retiendrai deux marqueurs majeurs de cette rupture intellectuelle : la singularité quelconque et l'image conversationnelle. La fin du dispositif politico subjectif des « classes sociales » nous a laissé orphelin d'un « nous ». La question $\mathrm{du}$ nous populaire est une question totalement ouverte aujourd'hui. Elle se donne comme une alternative entre des nous essentialisés et excluant que sont ceux de la xénophobie et 
de toutes les intolérances, et des nous inclusifs, mais éphémères, des « révolutions des places ", de Tien An men à Tahrir en passant par Taksim et Occupy Wall street.

5 Ces derniers Nous ne sont pas des " collectifs » au sens moderne, mais une capacité des singularités quelconques ${ }^{2}$ à construire du commun. Le lien dynamique du singulier et du commun l'emporte sur celui, moderne et aujourd'hui dépassé, de l'individuel et du collectif.

6 Le commun des singularités quelconques se construit dans le partage et dans le partage de l'image. C'est le deuxième marqueur. Le pictural turn proposé par W.J.T. Mitchell ${ }^{3}$ démultiplie les champs du partage du sensible ${ }^{4}$ dans ce domaine. L'image est au centre de la domination comme de sa contestation. La politique du sensible est à l'ordre du jour ${ }^{5}$.

7 L'image conversationnelle, celle partagée en live grâce au smartphone ${ }^{6}$ ou en léger différé sur Facebook ou Youtube est omniprésente dans les mobilisations les plus productrices de commun. Une nouvelle esthétique politique est en train de naître et de croître ${ }^{7}$. L'image partagée construit du commun sur l'événement. Le virtuel et la présence physique sont étroitement liés ${ }^{8}$, l'un se posant comme la preuve de l'autre et la présentation de sa subjectivité.

8 Si le développement numérique a complètement transformé nos usages des images, le passage du slogan à l'image sur les murs de nos villes est bien antérieur à ce basculement. Et sa place contemporaine dans la matérialité du partage ne se dément pas. En 2011 au Caire, les vidéos de la place Tahrir ont été complétées, prolongées par les murs de la rue Mohammed Mahmoud. Mais en l'absence de viralité, peut-on parler d'images conversationnelles ? Ces images ont le poids de la matérialité urbaine, elles sont toujours en situation et portent des énoncés visuels sur ces situations. Dans l'univers double de Matrix ${ }^{9}$, les graffitis sont du côté de la matérialité des corps et non de la circulation virtuelle des images de la matrice.

9 Vandales ou œuvres de commande, ces énoncés picturaux s'installent dans la ville. Ils en alimentent l'esthétique depuis plus d'un demi-siècle à rebours de toute fonctionnalité et le plus souvent de toute programmation. Mais ces énoncés ne peuvent s'inscrire nulle part ailleurs. Intempestifs, ils sont aussi indélocalisables. C'est pourquoi nous devons lire ces énoncés picturaux comme un langage contemporain dans leurs situations urbaines, comme le langage singulier de communs urbains en travail ${ }^{10}$. 


\section{Murs blancs peuples muets : la bataille des murs}

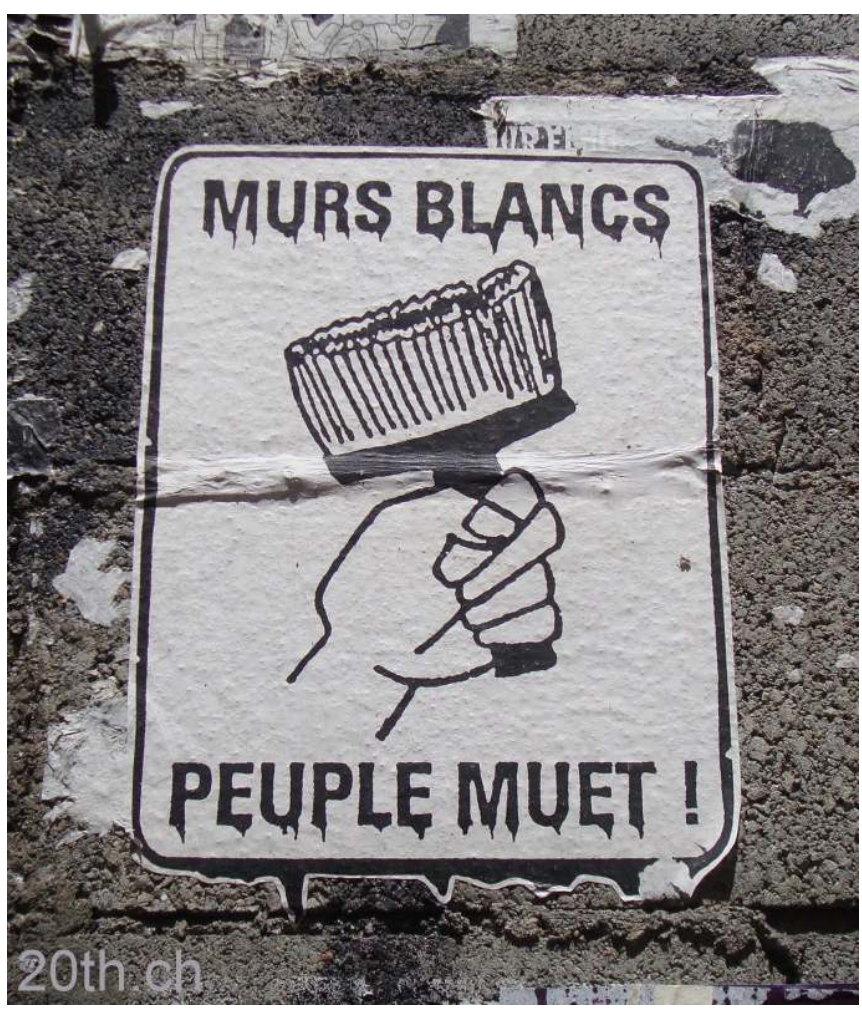

Le slogan « Murs blancs peuple muet » et l'image ${ }^{11}$ qui le porte nous viennent sans doute de Mai 1968. C'est devenu une rumeur internationale qui se décline dans plusieurs langues notamment en espagnol: Muros brancos, povo mudo ou Paredes brancas, povo mudo.

Certes, son esthétique et la symbolique de l'outil sont un peu d'un autre temps. On est loin des bombes aérosols contemporaines, voire des extincteurs de peinture de Kidult. Mais la pérennité du slogan indique bien le maintien d'une «bataille des murs» qui touche autant les dictatures que les démocraties. Cette bataille a pour protagoniste l'ordre urbain qu'il soit institutionnel ou marchand et face à lui un adversaire insaisissable qui introduit sans cesse un dissensus, travaille le sens, exhibe une subjectivité de la ville que cet ordre rend invisible. Les murs parlent d'abord la langue de la polémique. 


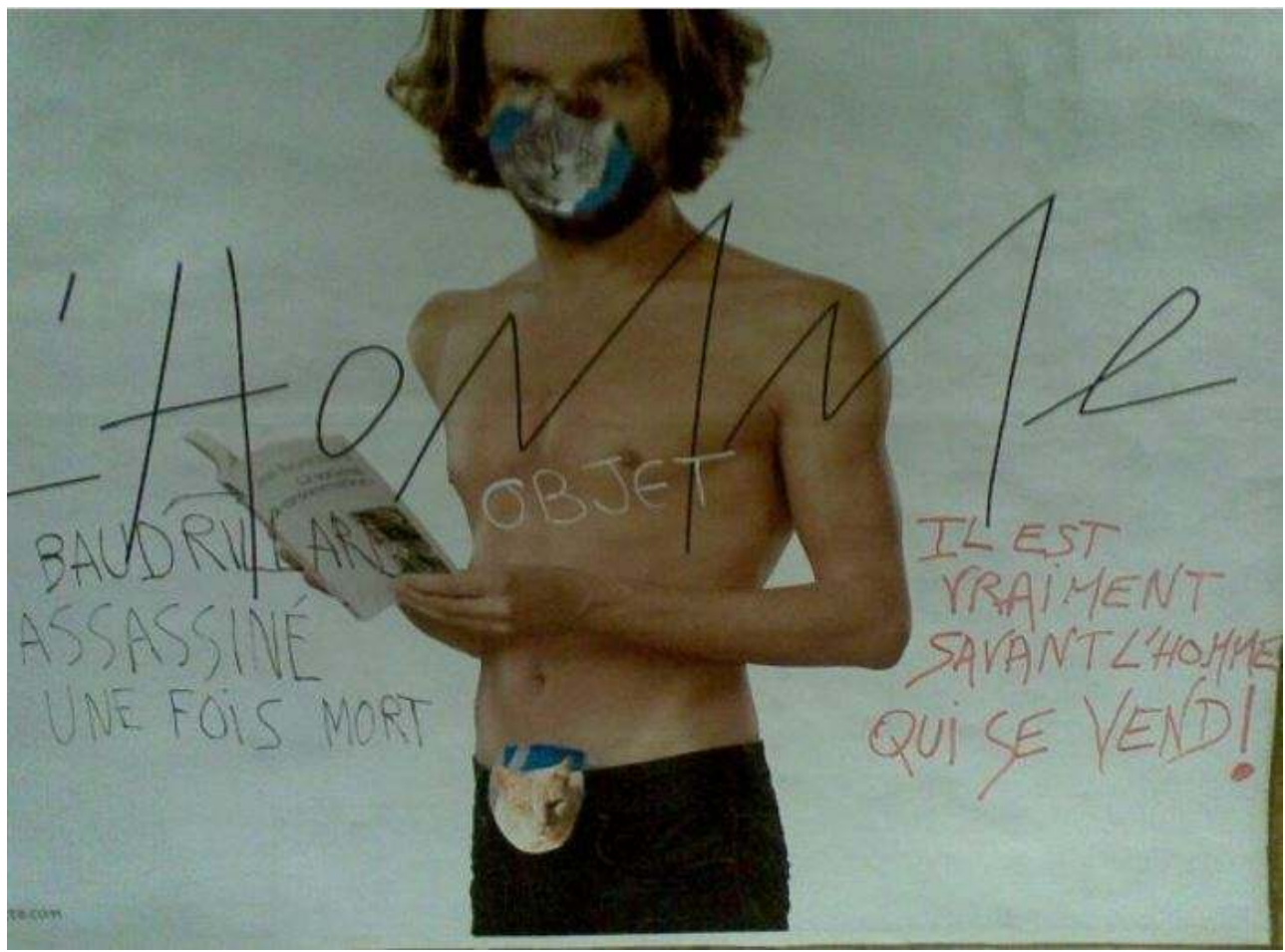

Métro Pont Marie, 2008. Photo Alain Bertho

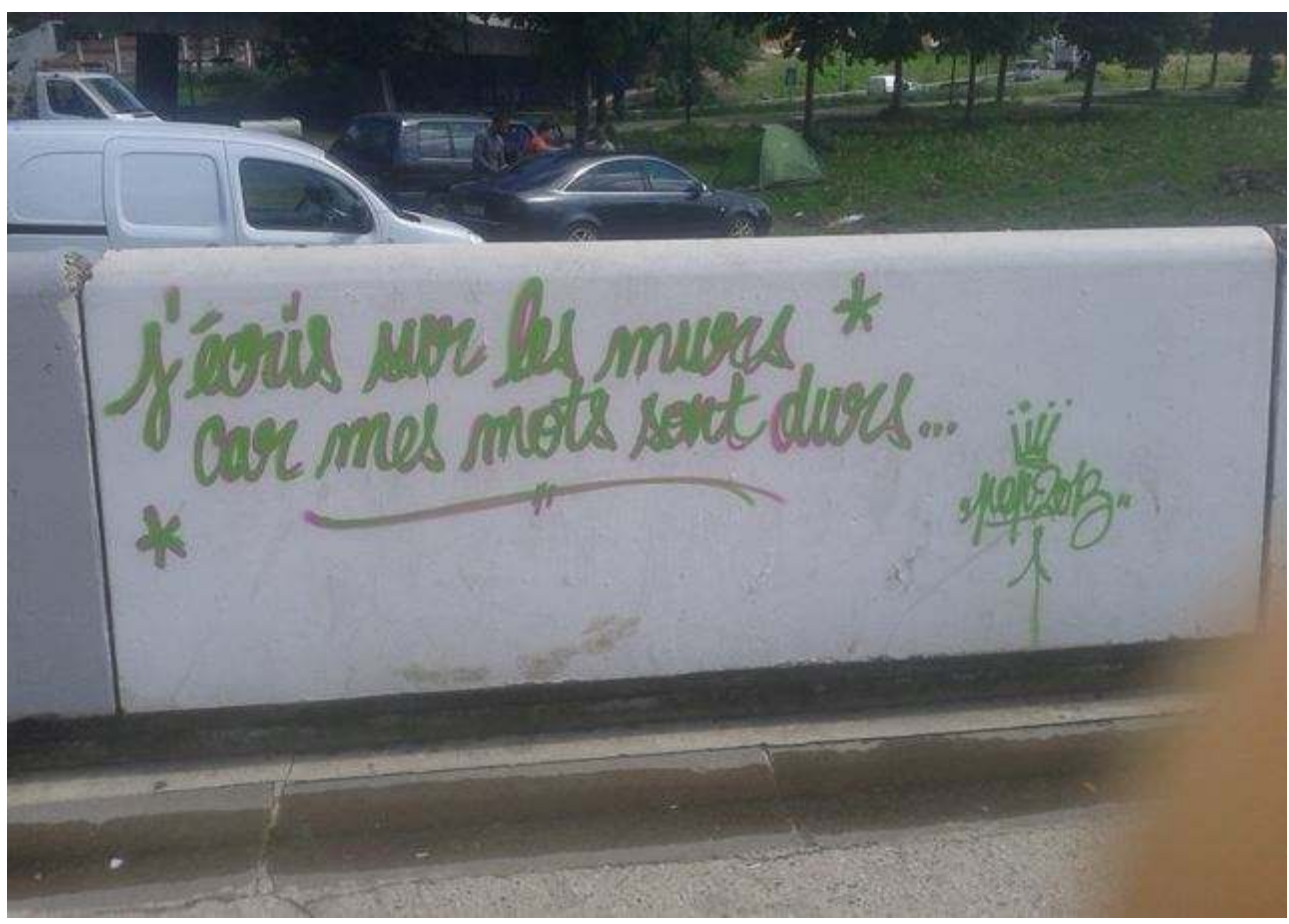

Saint-Denis 2014. Photo Alain Bertho 


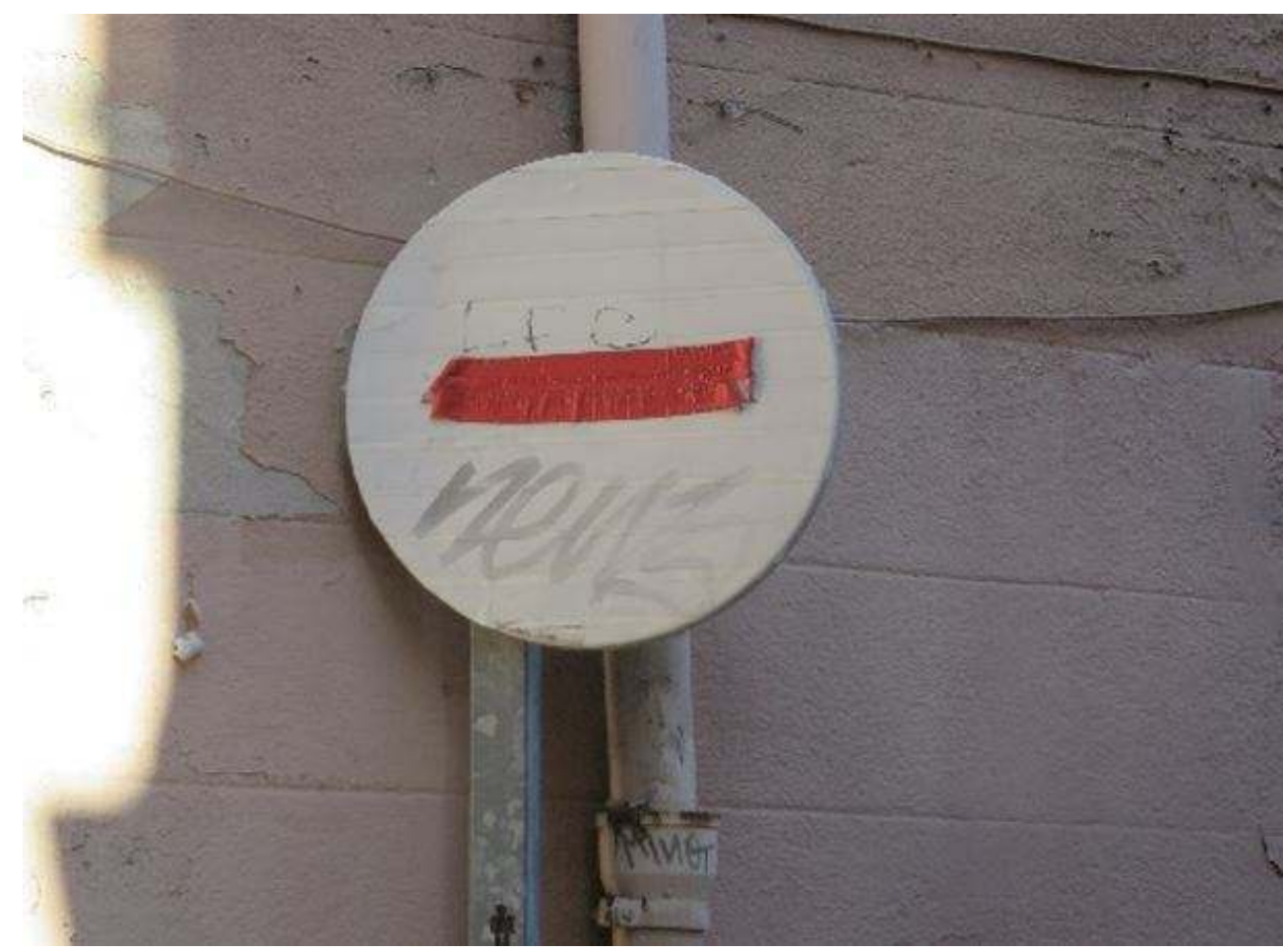

Marseille, quartier du panier 2015. Photo Alain Bertho

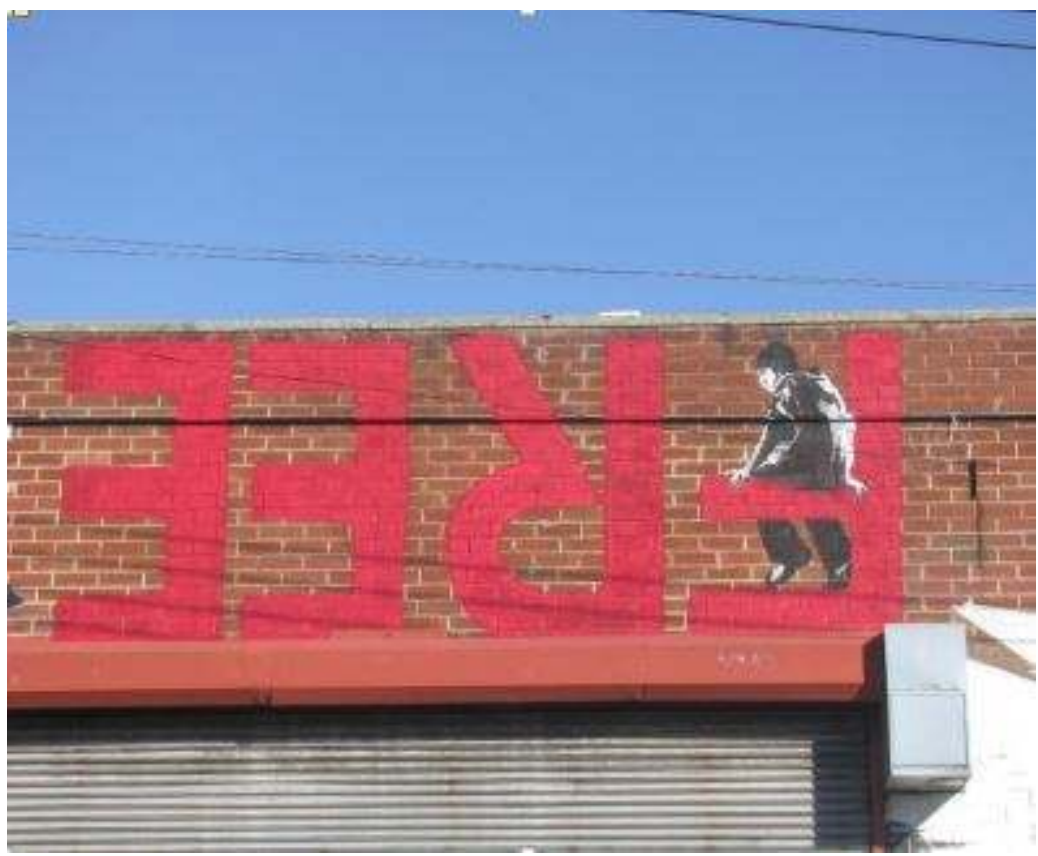

New York, Busch wick collective, 2013. Photo Alain Bertho

12 La nature de ce dissensus, sur lequel il va nous falloir revenir, peut être diverse. L'ordre urbain peut même séparer le bon du mauvais. Pedro Araya dans une thèse sur les murs de Santiago soutenue en 2015, raconte comment après la dictature, un débat s'est ouvert au Parlement sur la réglementation des murs: pouvait-on interdire les graffitis mais autoriser les affiches électorales ${ }^{12}$ ?

13 Comme si la polémique ouverte par ce qui s'affiche et s'écrit était légitime lorsqu'elle était structurée comme une représentation du social et du politique et illégitime 
lorsqu'elle était dans la seule présentation de soi. Les murs sont bien les mêmes, les situations urbaines aussi. Mais ce qu'on y affiche ne travaille pas la situation de la même façon, n'est pas partagé de la même façon ni par les mêmes passants. L'affichage politique relève d'une conflictualité normée, mobilisant des représentations et des énoncés collectifs et contribuant à produire de l'ordre. Les graffitis n'introduisent pas du conflit mais du dissensus, ils ne portent pas de collectif mais du singulier et de l'éventuel partage.

14 Ces deux régimes sont distincts et pour une part successifs. La vague du graff puis du Street art depuis un demi-siècle n'a pas seulement marqué l'esthétique du contemporain de nos villes. Elle y a introduit une nouvelle esthétique politique nécessitant d'autres protocoles de lecture et d'analyse.

\section{Portaits des collectifs : les mineurs de ladrecht}

15 Nous avons parlé de la modernité, il nous faut y faire un retour. Les fresques murales ne sont en effet pas nouvelles. Elles ont des lieux emblématiques: Orgosolo en Sardaigne ${ }^{13}$, Belfast ${ }^{14}$, les murales d'Amérique Latine ${ }^{15}$. Ces fresques ont en commun de donner à voir les symboles d'un collectif de mobilisations pré établies, de parler le langage de l'épopée dans des conflits très identifiables : conflits de classe ou conflit à caractère national.

16 L'acte de peindre y a pris le sens d'un engagement indexé à d'autres références, d'autres symboliques qui faisaient dispositif culturel et subjectif.

17 Une fresque de ce type a accompagné au cœur des Cévennes, les derniers mois de la mobilisation des mineurs de Ladrecht contre la fermeture du puit Destival à Saint-Martin de Valguargue ${ }^{16}$.

À l'aube des années 1980, cette œuvre monumentale est due à l'engagement d'un collectif d'artistes, l'Union régionale des artistes plasticiens ${ }^{17}$, soutenu par d'autres créateurs régionaux tels Jean Pierre Chabrol :

Voilà, cette fameuse équipe est arrivée pour faire de la peinture avec des tonneaux colorés, des échelles, des pinceaux de bâtiment, et surtout des mains intelligentes, larges, fortes comme des pognes ouvrières. (...) Voyez, ce mur chante, ce mur se bat. (...) A force de talent, ce mur enchanté par la fresque est entré dans le paysage, et dans l'histoire prolétarienne des rebelles ${ }^{18}$. 

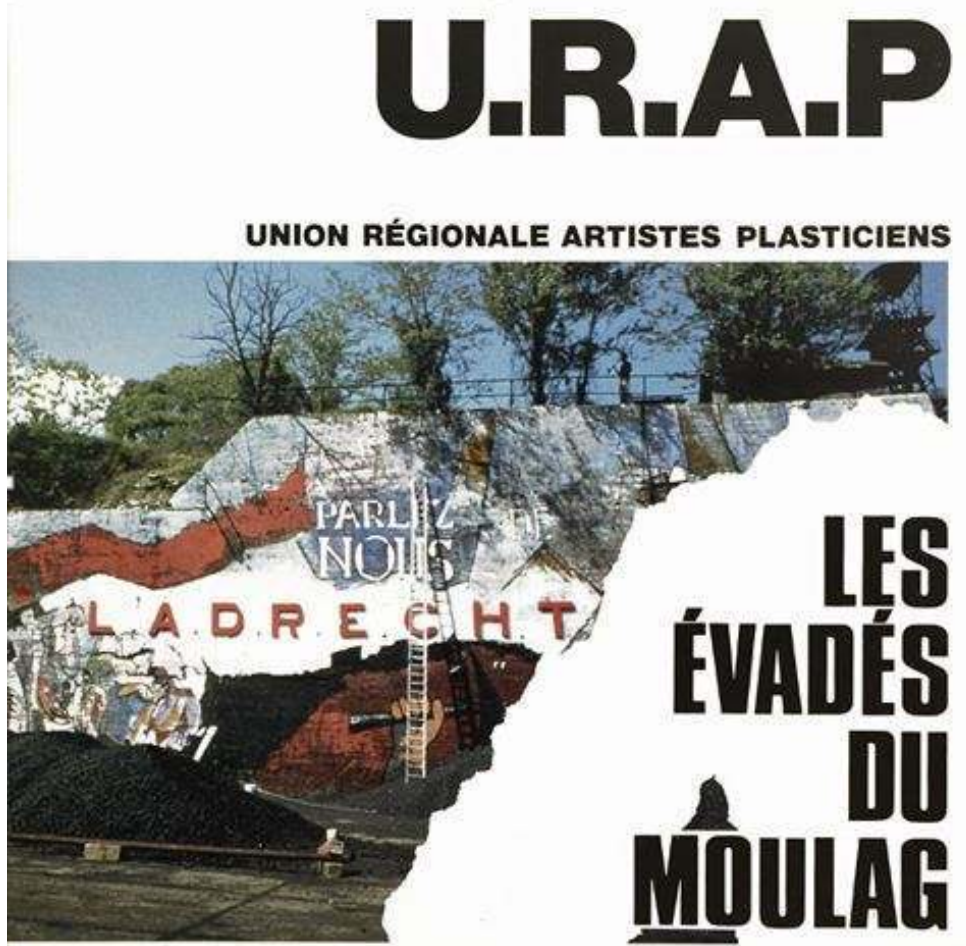

19 Symboles monumentaux du travail ouvrier et de la mine, poing levé, piolet brandi, rouge de l'étoffe : le répertoire visuel est convenu, il s'inscrit dans une histoire, mobilise des références, lance un appel. L'adresse qui surplombe la fresque est explicite : «Parlez de nous!» Ce Nous est sans ambiguité. Il est la déclinaison locale d'un Nous populaire préexistant, politique et référencé.

Plus de trente ans ont passé. L'époque est close et cette histoire prolétarienne semble oubliée. Il reste du mur une photo dans un coin du musée du Puit Ricard à la GrandCombe. L'original a été découpé et saccagé pour installer un bassin de rétention. 


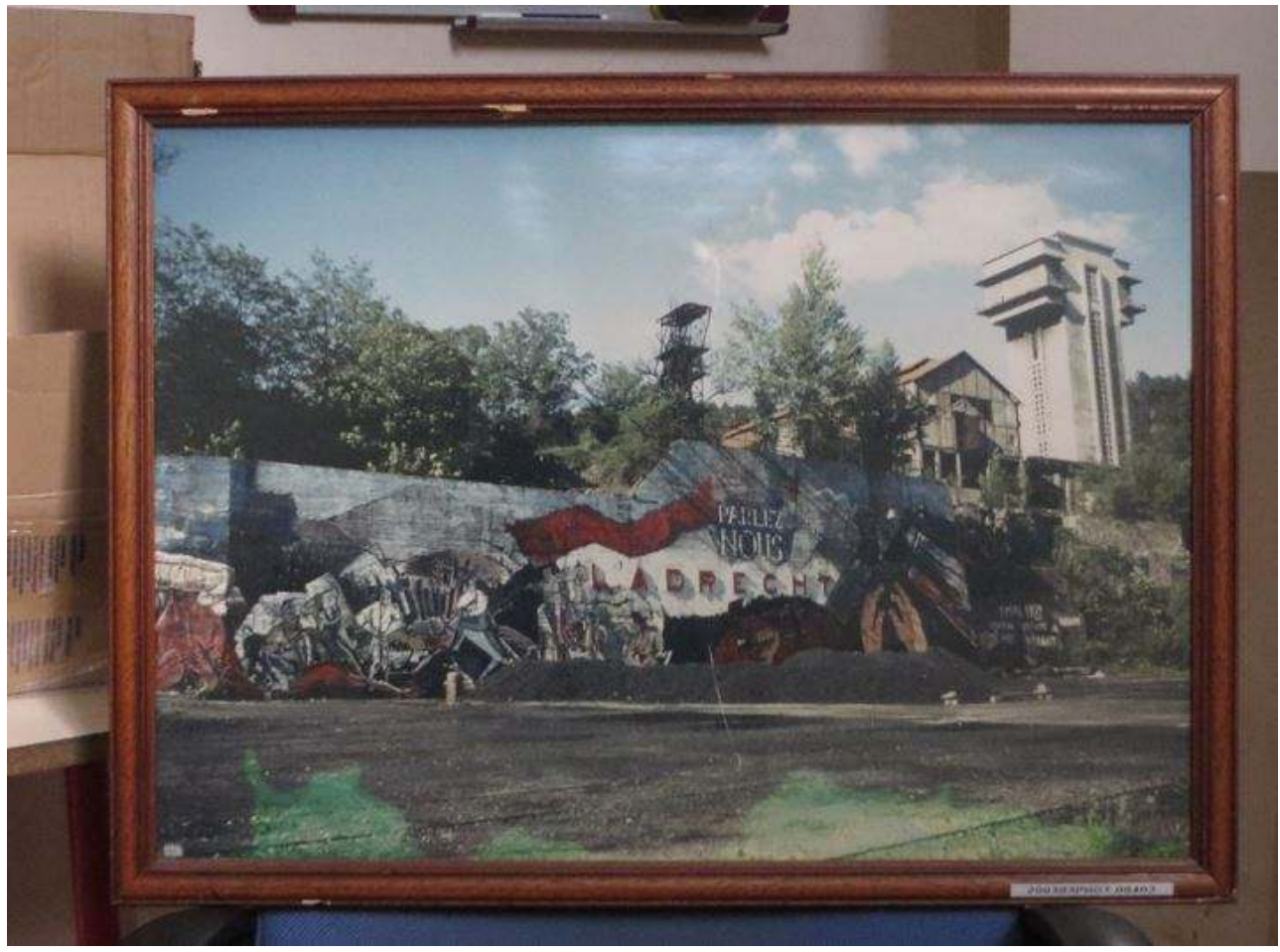

Photo Alain Bertho septembre 2015. Musée du Puit Ricard

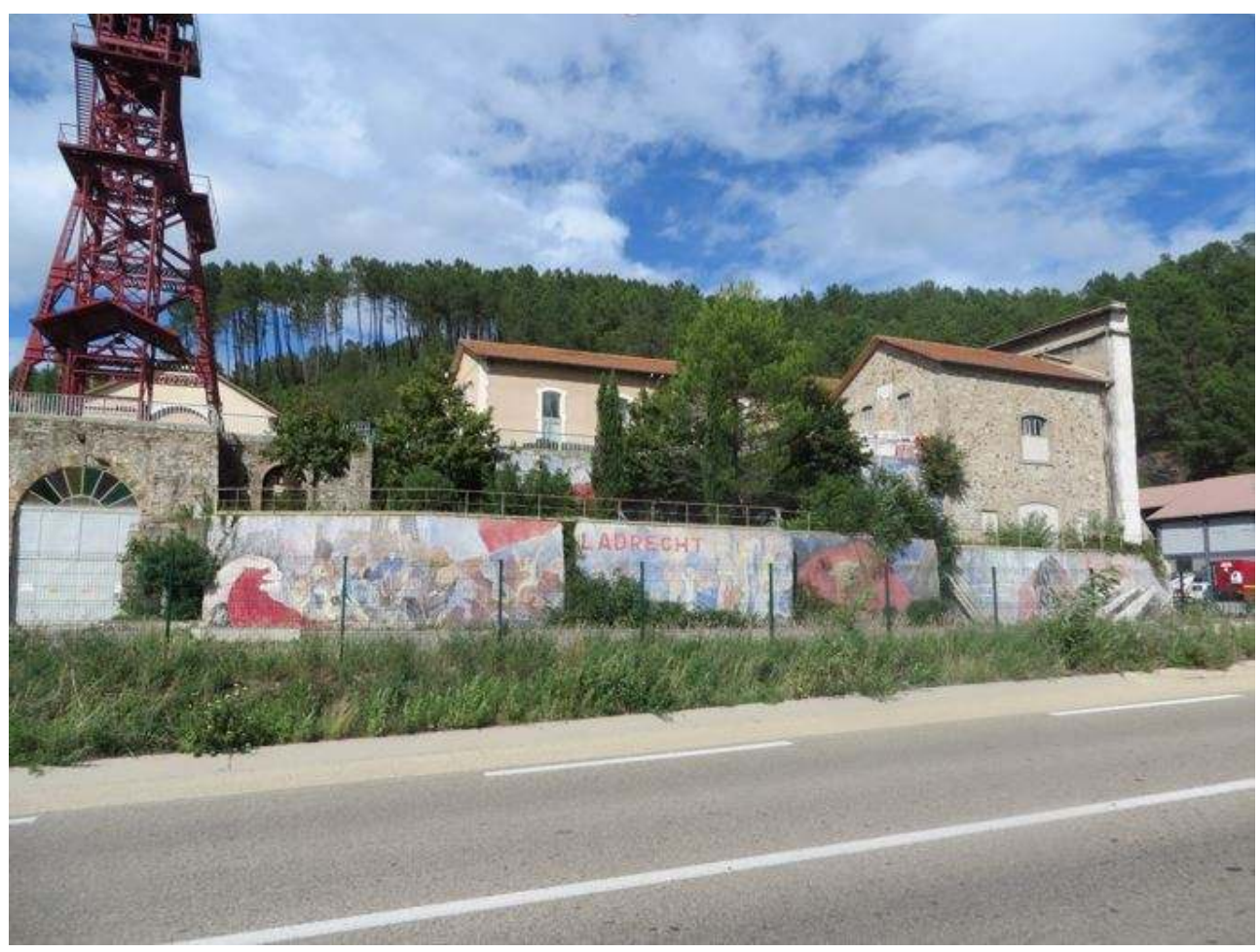

Photo Alain Bertho septembre 2015 La fresque aujourd'hui

21 Quant au souvenir des luttes et de la conflictualité qui avait façonné cette société locale, il a bien disparu des murs de Saint-Martin de Valgargue où s'affiche un consensus de mémoire minière sans conflit. 


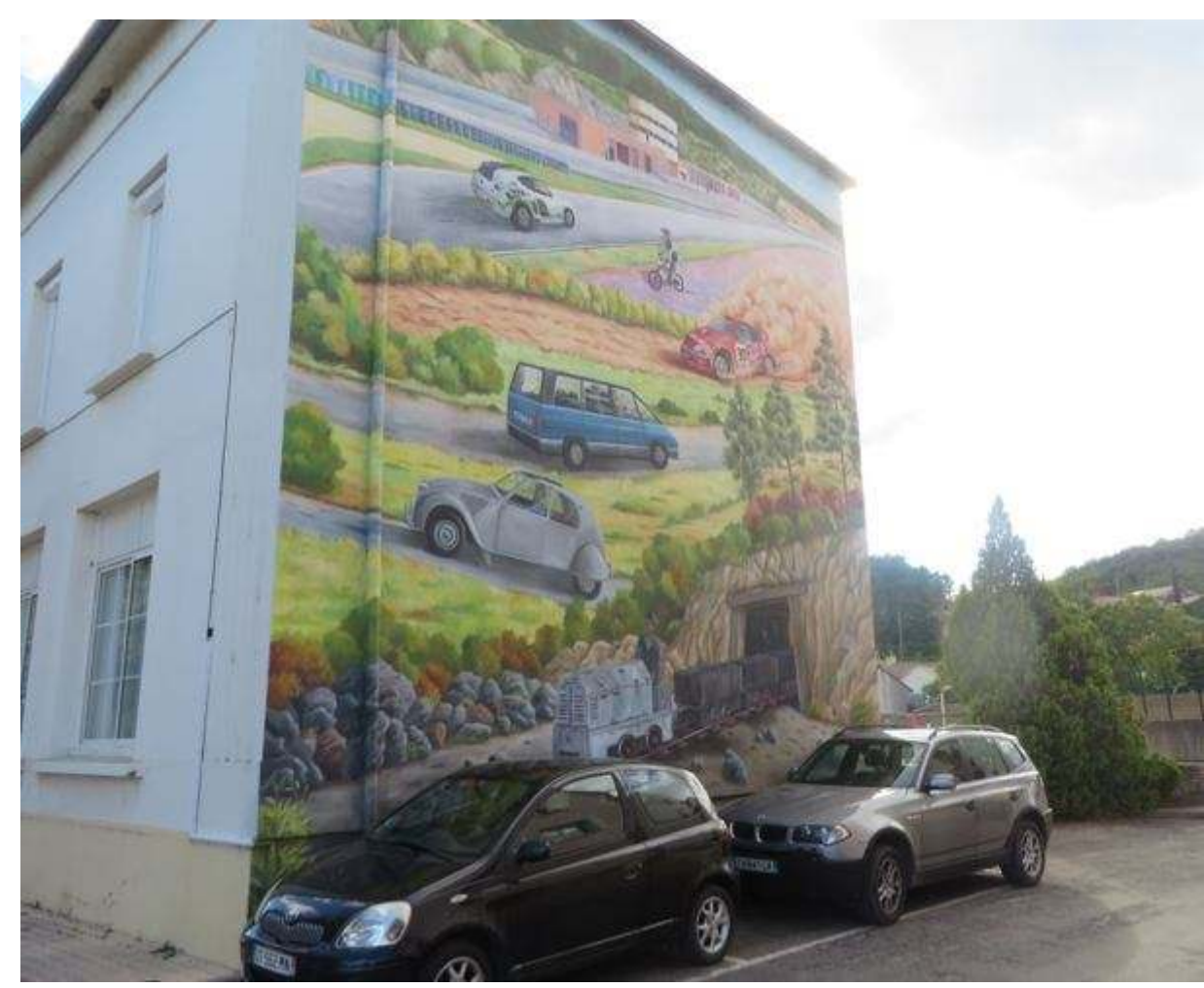

Photo Alain Bertho septembre 2015

\section{5-2011 portraits des singularités}

Conflits et identités de classes s'achèvent avec le siècle. Il nous faut d'autres mots pour parler des «nous » qui émergent, des mobilisations qui les constituent. D'autres mots pour parler des affrontements matériels ou symboliques qui s'ouvrent. D'autres mots et d'autres protocoles pour lire des images dont l'omniprésence est une des marques de ce temps.

D'autres dissensus se sont ouverts pour lesquels la constitution d'un sujet subjectif, d'un « Nous» est un enjeu récurrent qui articule toujours le singulier et le commun. Il est frappant de voir comment certains street artistes s'inscrivent dans cette nouveauté du temps et peuvent en parler par l'image mieux et avant tous les discours convenus.

L'intervention du très jeune JR dans sa cité des Bosquets à Montfermeil dès 2004 dans le projet qui deviendra « Portrait d'une génération » est significatif à cet égard. 


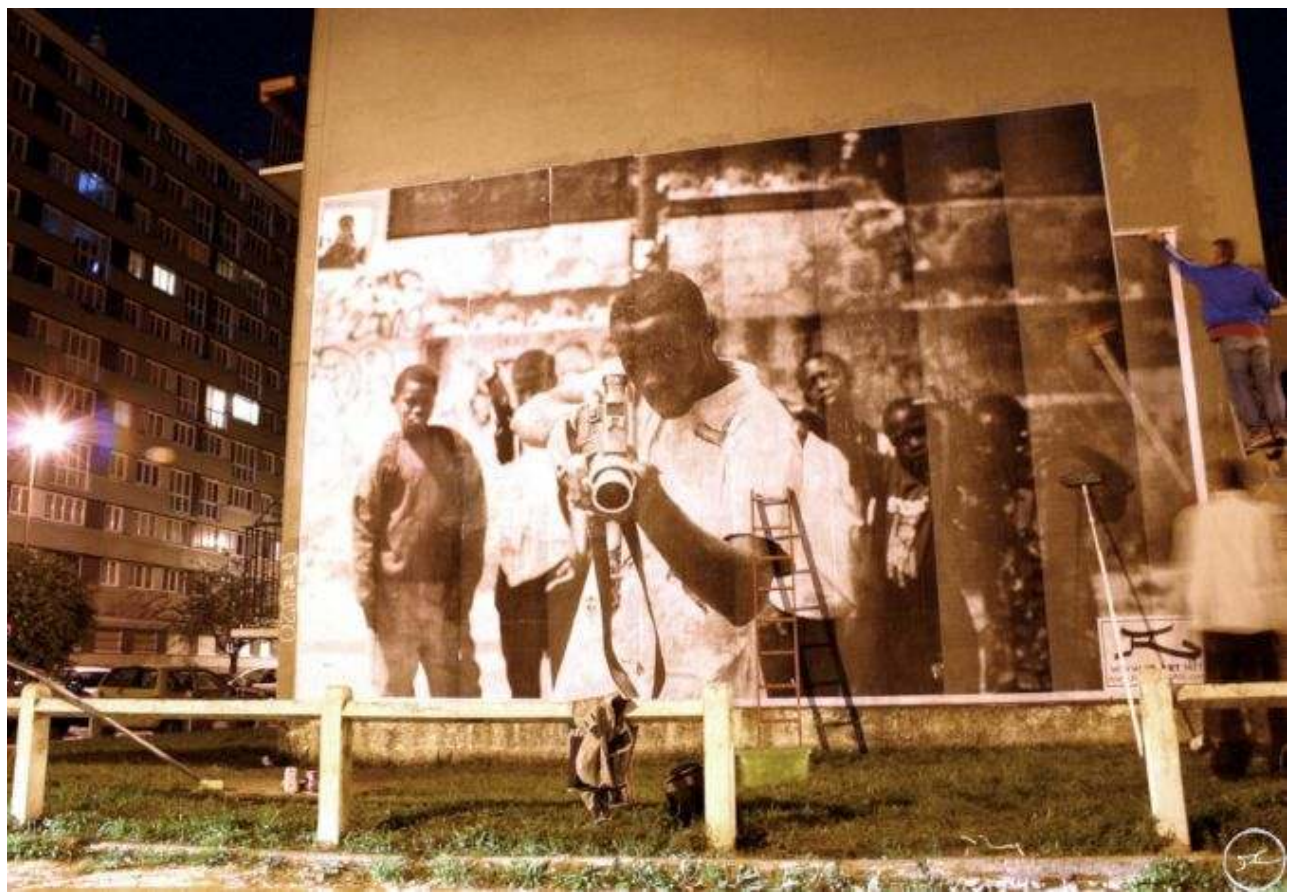

Braquage, Ladj Ly par JR, Les Bosquets, Montfermeil, 2004 http://www.jr-art.net/fr/projets/portraitdune-generation

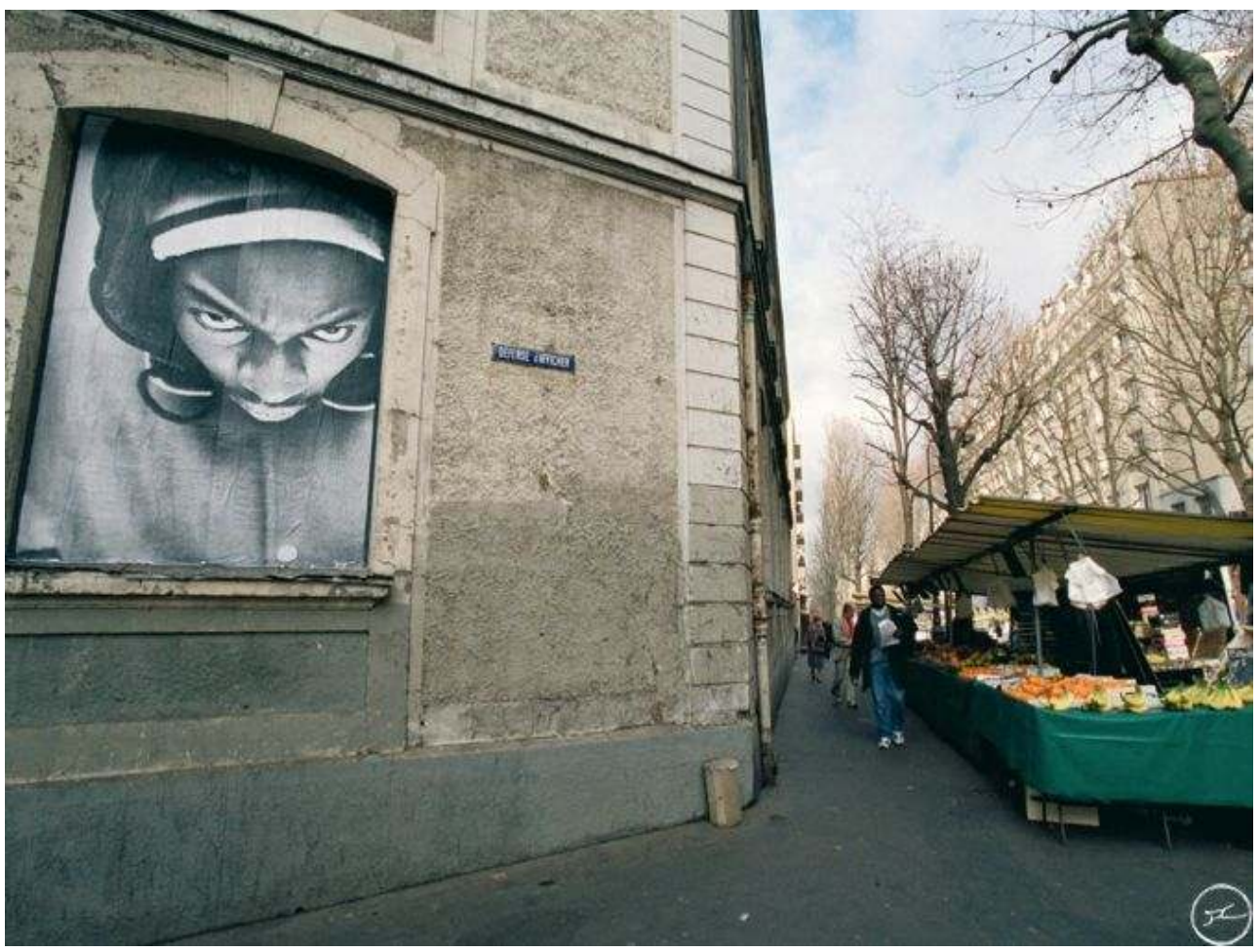

JR Portrait d'une Génération Byron, Paris, 20ème arrondissement, 2004 http://www.jr-art.net/fr/projets/ portrait-dune-generation

Ouvert par le fameux « Braquage » avec Ladj Ly, il se prolonge après les émeutes de 2005 avec ces portraits géants en gros plans qui s'affichent bien au-delà de Clichy Montfermeil. JR met tout simplement des visages et de la singularité sur une colère qui fut celle de la nuit et des flammes ${ }^{19}$. Il identifie les invisibles, ceux que l'institution ne compte jamais pour un. Sans texte, par la seule force de leur énoncé pictural, ces images ne 
« représentent » rien ni personnes contrairement aux murales du XXe siècle. Elles sont un dispositif de présentation sans médiation épique ou discursive ancré dans le quartier mais qui va s'autoriser une délocalisation matérielle et symbolique. Quand les incendies de voiture sont restés cantonnés aux cités, les portraits de JR s'imposent sur d'autres murs et notamment à Paris.

Ces portraits de singularités quelconques dont le principe est repris dans «Inside Out » obtiennent un certain succès lors de la révolution tunisienne de 2011. C'est dans cette même Tunisie en ébullition que Bilal Berreni ${ }^{20}$ expose ses silhouettes anonymes découpées.

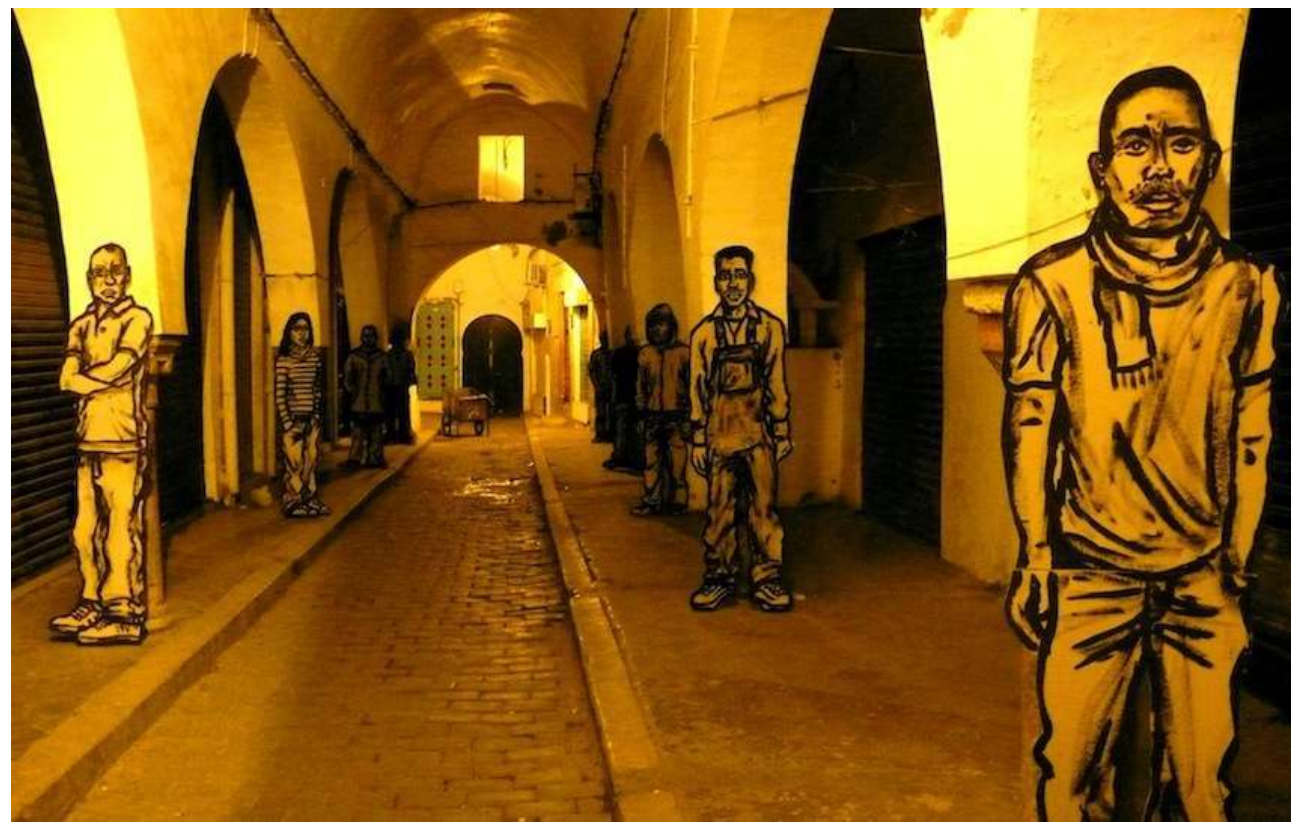

Bilal Berreni en Tunisie. http://www.lumieresdelaville.net/2014/12/22/presidentielles-tunisie-le-streetart-comme-un-hommage-aux-martyrs-de-la-revolution/ 


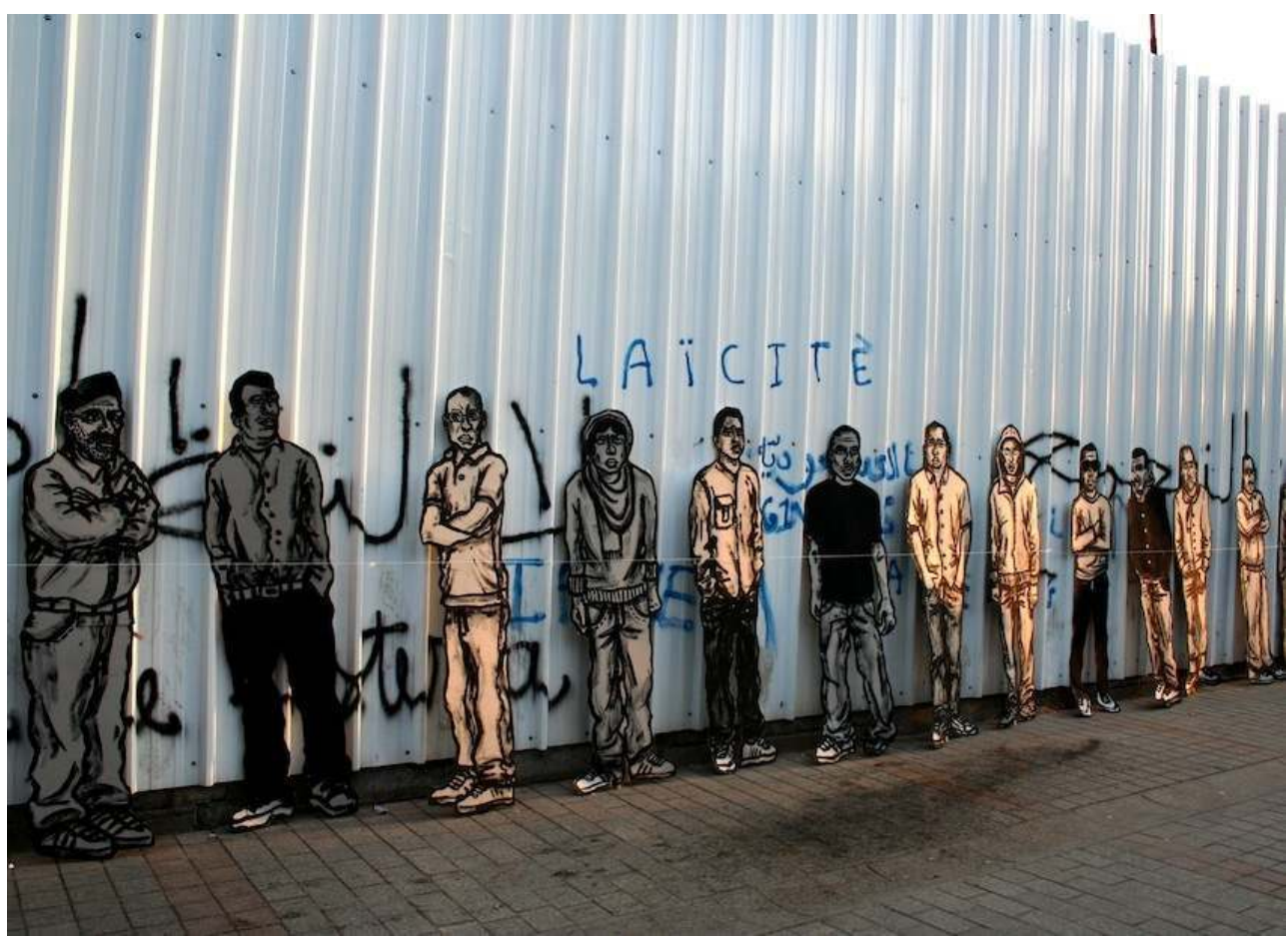

Les « martyrs », le long de l'avenue Bourguiba à Tunis. Zoo Project. http://

www.lumieresdelaville.net/2014/12/22/presidentielles-tunisie-le-street-art-comme-un-hommage-auxmartyrs-de-la-revolution/

Ces deux artistes travaillent à rendre visible ensemble des singularités sans les confondre dans une foule massifiée. Ils nous dessinent une figure du dissensus constituée par l'émergence même de ces singularités invisibles dans l'espace commun.

Là où le collectif politique du XXème siècle portait un dissensus social et politique globalisé, c'est l'émergence de ces visages singuliers qui est la matière même du conflit, l'objet du scandale, le retour du refoulé urbain social et politique. Avec «Women are heroes ", JR n'avait-il pas donné des yeux immenses aux femmes de la favela Providentia, permettant à ces habitantes invisibles d'imposer leur regard sur le reste de la ville de Rio de Janeiro?

29 Ces artistes et d'autres moins connus contribuent ainsi à des autoportraits de mobilisations dont nous avons eu des figures emblématiques sur les murs grecs de $2012^{21}$, de Tunisie ${ }^{22}$ et d'Egypte ${ }^{23}$ en 2011. L'Europe boulimique d'euros à Athènes, les silhouettes de Bilal à Tunis, les mères et les martyrs de la rue Mohammad Mahmoud ont marqué et exprimé la singularité locale des mobilisations.

\section{Déchirer la ville}

30 Avec les Writers, le surgissement des singularités n'attend pas les émeutes ou les mobilisations de 2011. « Déchirer un mur, brûler un train, tuer une rue » : il faut peut-être prendre le vocabulaire vandale au pied de la lettre. Ce sont des traces singulières qui ouvrent des brèches visibles, ostentatoires dans l'ordonnancement urbain ${ }^{24}$. 

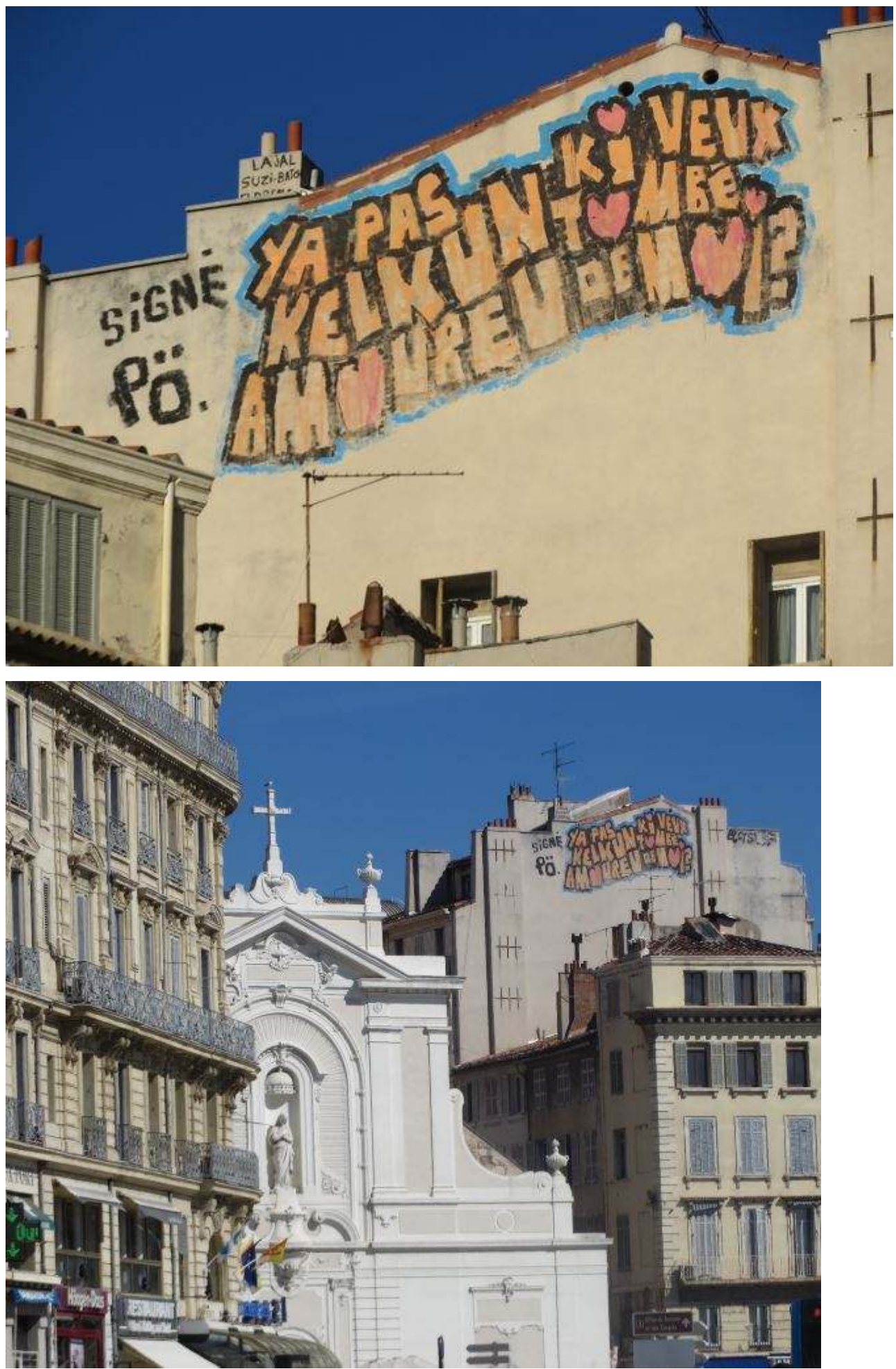

S'exhiber sur le Vieux port de Marseille photo Alain Bertho. Septembre 2015

Elles en travaillent les plaies ouvertes dans les friches industrielles, les terrains vagues, les palissades. Elles marquent de façon ostentatoire les réseaux : les trains, les métros, les autoroutes urbaines. Elles lancent des défis crâneurs aux verticalités inaccessibles. Elles s'accrochent aux portes et aux rideaux des commerces. Parfois opportunistes, parfois provocatrices, ces interventions polémiquent toujours avec l'ordre de la ville sans 
toutefois donner à voir le moindre ordre alternatif. Anonymes et invisibles, nous sommes là, indéracinables. Tel semble le seul message commun de ces interventions.

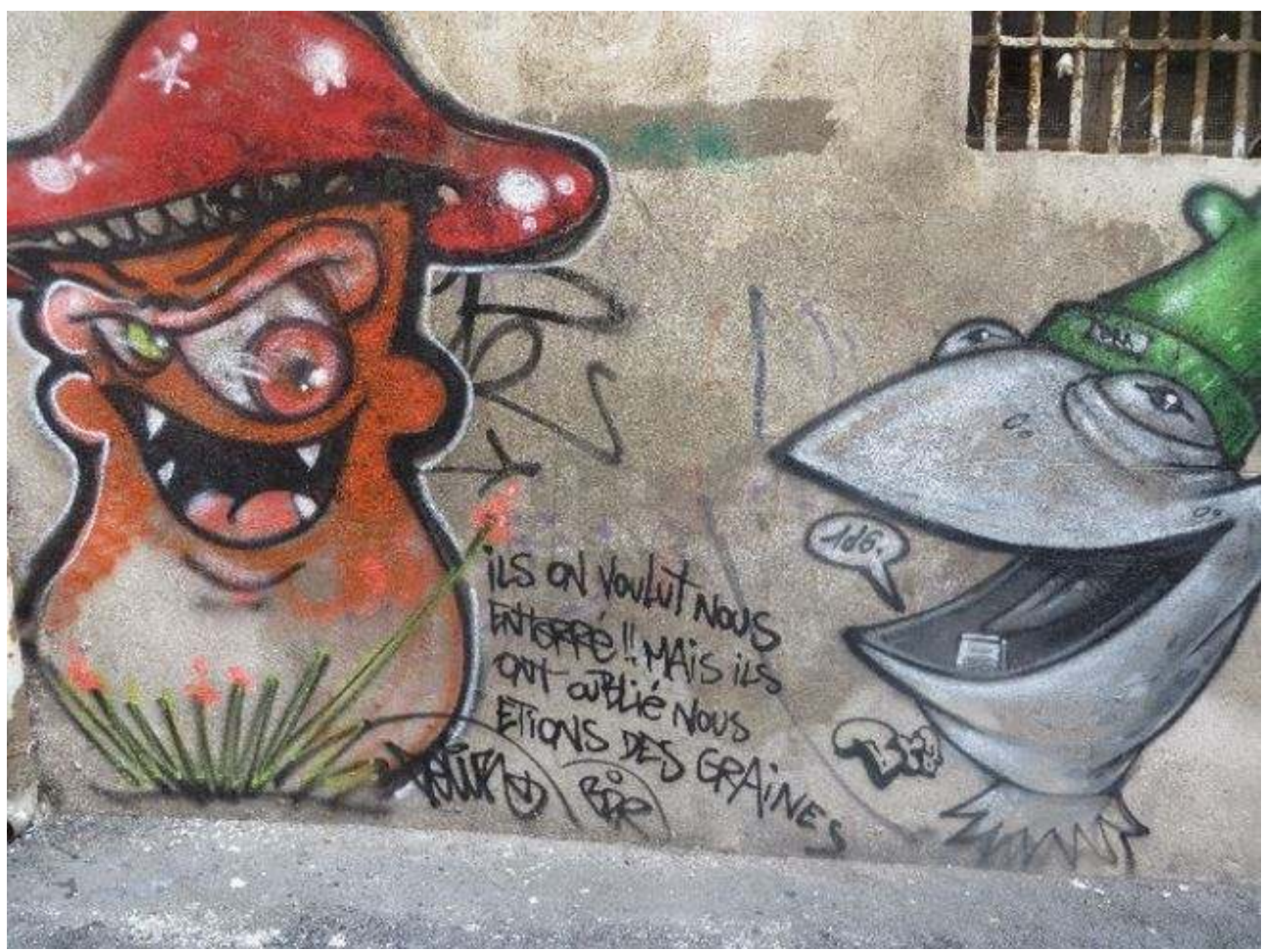

Marseille septembre 2015. Photo Alain Bertho

32 C'est notamment dans sa capacité à aller plus loin et plus explicitement dans le dévoilement et la polémique que Banksy marque son temps. Décalés, ironiques ses énoncés picturaux sont directement partageables. Ils mobilisent un en-commun culturel élaboré et circulent bien au-delà des situations urbaines qui les a vus émerger. ${ }^{25}$ Outre la prise de parti explicite contre la vidéo surveillance, le mur israélien ou la répression des jeux enfantins, on voit par exemple convoquer des références à Guatanamo, aux peintures rupestres comme à la Joconde, à une photo icone de la guerre du Vietnam ou au salut au drapeau... 

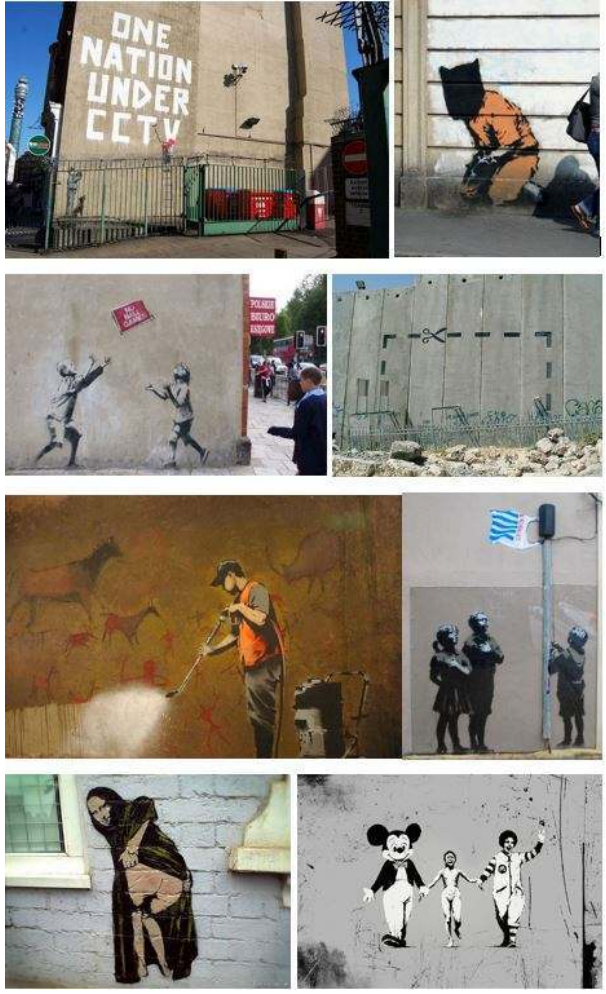

http://soocurious.com/fr/80-oeuvres-de-lartiste-banksy-qui-vous-feront-voir-le-monde-dune-autrefacon/?utm_source=feedburner\&utm_medium=feed\&utm_campaign=Feed\%3A+DailyGeekShow+\% 28Daily+Geek+Show\%29

\section{Des autoportraits urbains?}

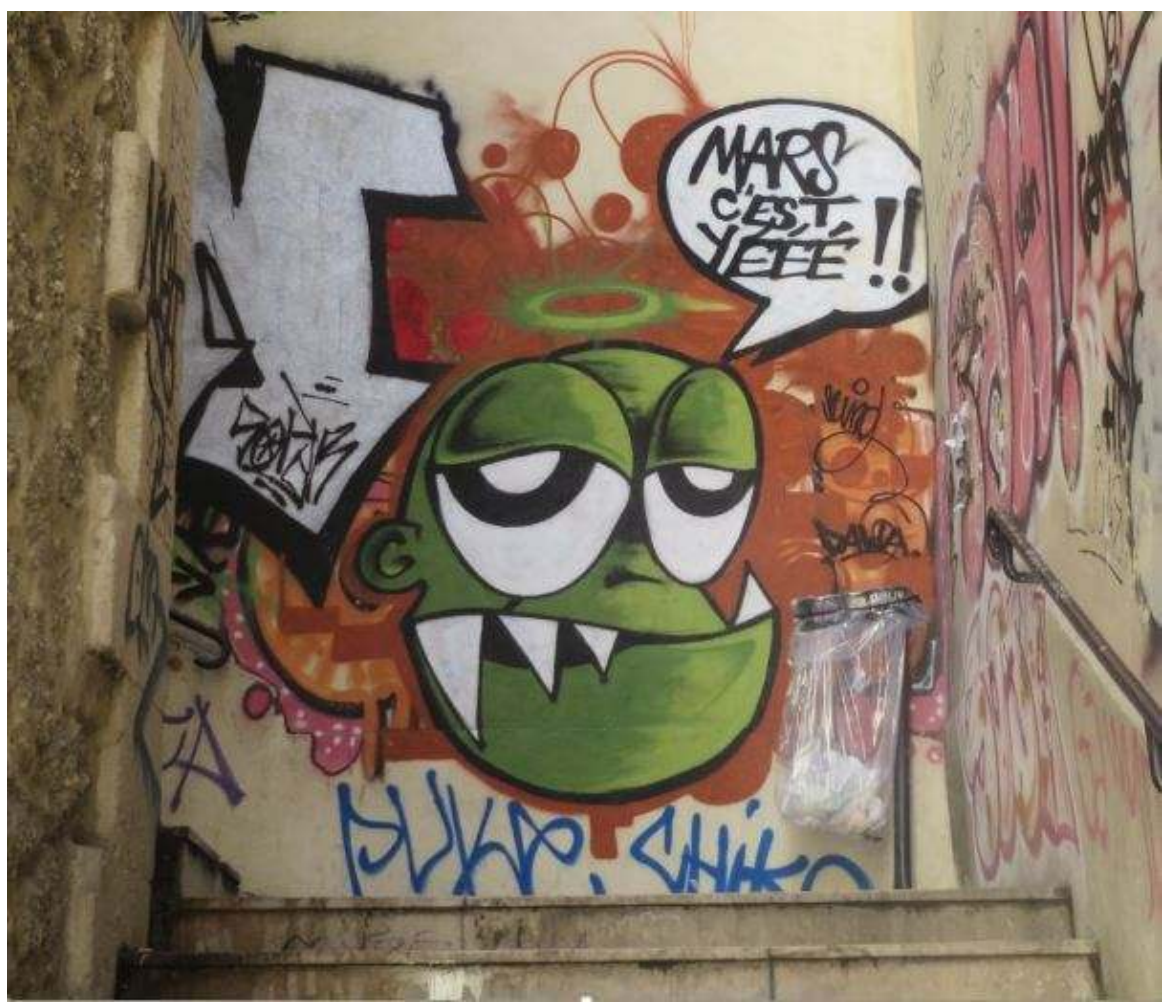

Marseille 2015. Alain Bertho 
Graffiti et Street art constituent aujourd'hui un ensemble de pratiques et une esthétique à l'échelle des villes du monde. Ils sont un élément important, à la fois matériel et localisé, de «l'ethnoscape » contemporain dessiné par Arjun Appadurai ${ }^{26}$.

Cette mondialisation esthétique qui relie les villes et leurs habitants par-delà les océans et les langues produit aussi de l'identité et de la différence ${ }^{27}$, pour être plus précis, de la singularité. Et cette singularité est localisée car les murs battent au rythme du cœur des villes. Ils affichent l'autoportrait de la ville réelle. Ils identifient les lieux vivants comme les monuments identifient le patrimoine.

35 En Europe par exemple les murs de Berlin ne sont pas ceux de Palerme ou de Naples. Le défunt Tacheles et East Side identifiaient la trace sans fin qui marque la ville de Berlin, une ouverture sur l'absentement de l'histoire paradoxalement patrimonialisé. La Pieuvre et ses monstres rodent sur Palerme dans l'indifférence institutionnelle et vaticane, les faux semblants et l'omertà. Les monstres qui peuplent Naples sont plus divers et moins menaçants dans un centre-ville qui semble dédié à ses murs et à ceux qui les hantent.

De Tanger à Dakar, l'Afrique s'écarte de l'Europe et du poids du pouvoir. Le port de Tanger regarde l'Espagne et l'Europe. L'ombre du pouvoir (le MAHZEN) plane sur les murs de Rabat, entre affiches déchirées, les writers furtifs et les artistes subventionnés par la poste qu'on retrouve au Musée d'art contemporain. L'Afrique perce déjà à Essaouira et vient brouiller la trace coloniale à Dakar entre la francophonie des writers (très peu d'inscription en wolof) et les mystérieux visages qui regardent les passants.

37 La capitale Carioca, quant à elle, a autant de visages que de quartiers, foisonnante, musicale et brutale. Les murales brassent les styles et les identités.

Berlin

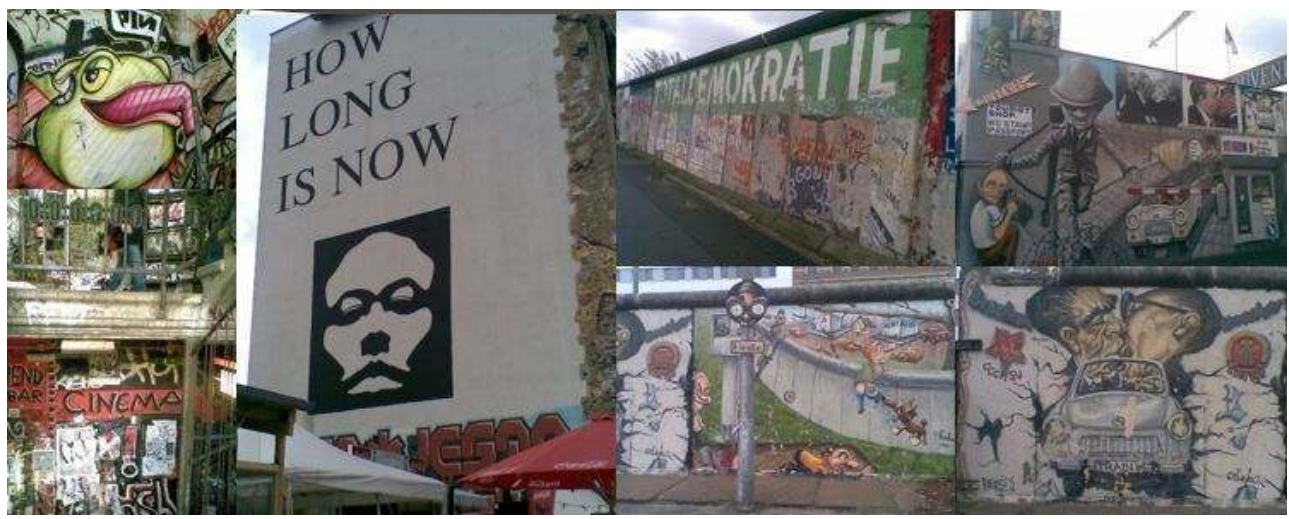

Berlin 2007. Photos Alain Bertho 


\section{Palerme}

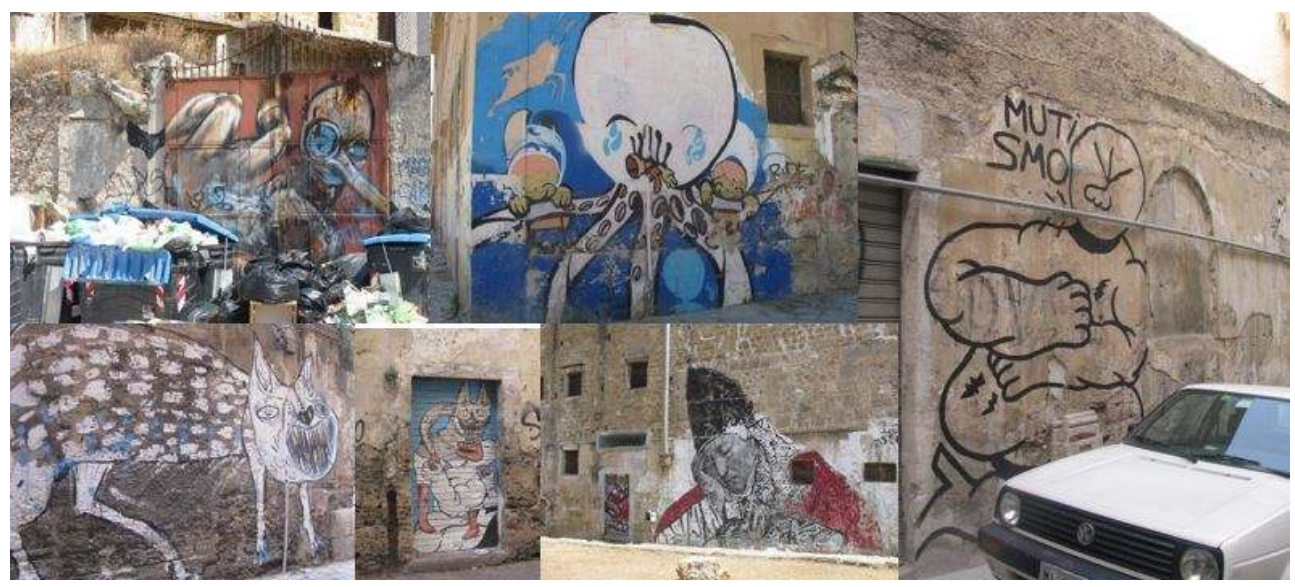

Palerme 2012. Photos Alain Bertho

\section{Naples}

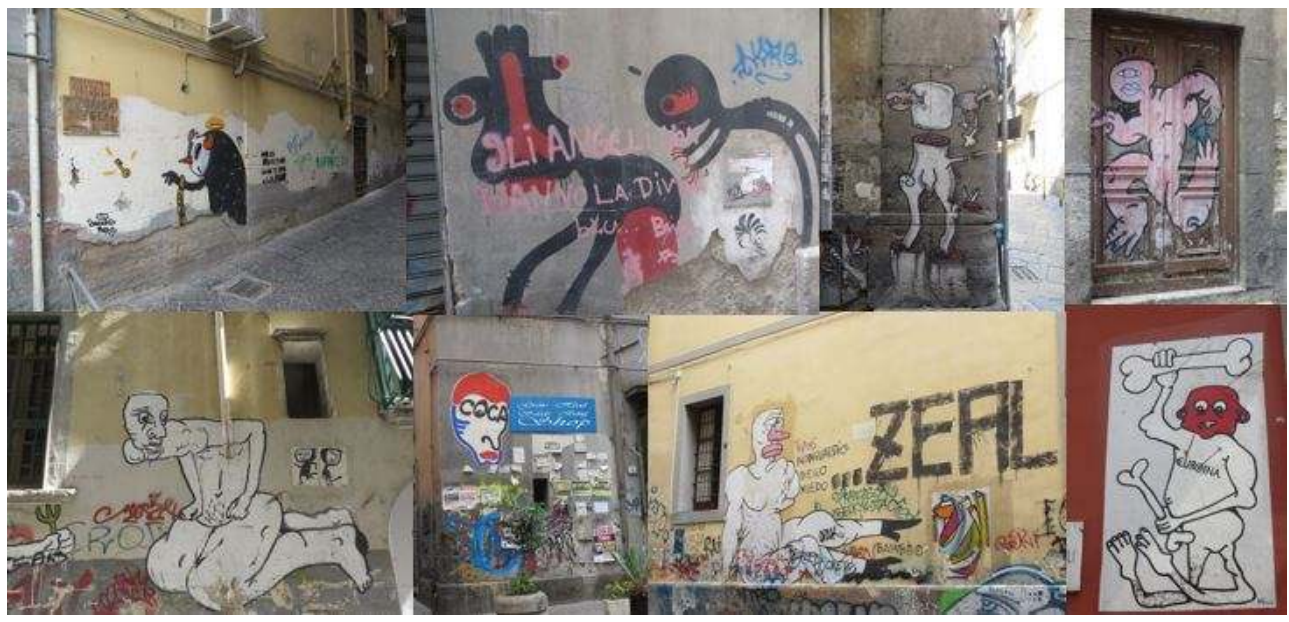

Naples 2015. Photos Alain Bertho 
Tanger

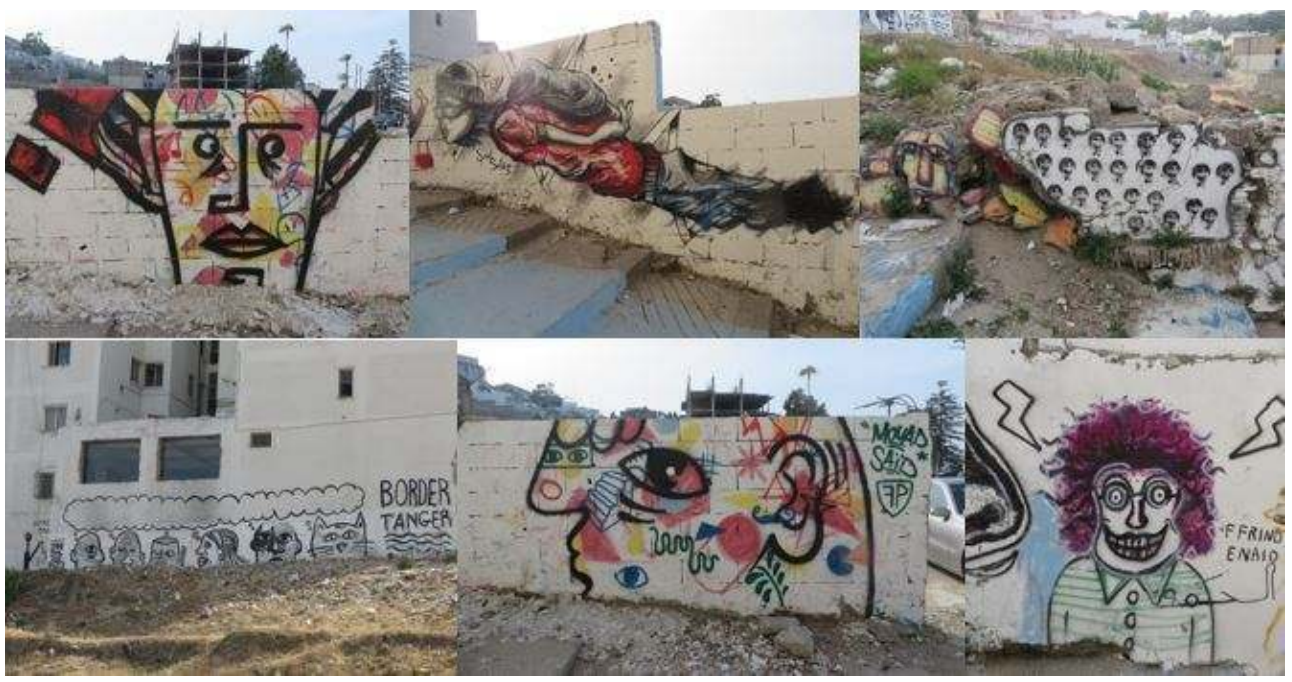

Tanger 2015. Photos Alain Bertho

\section{Rabat}

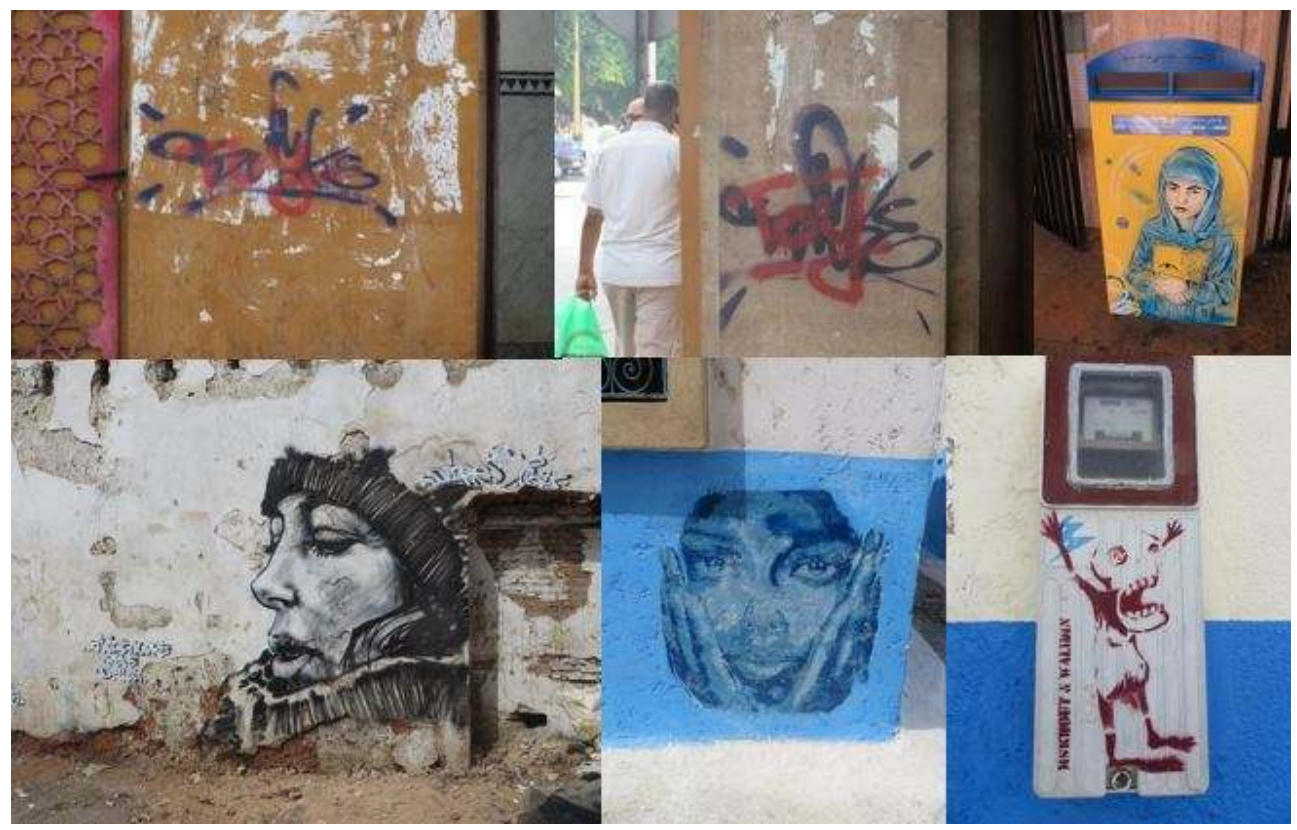

Rabat 2015 ; Photo Alain Bertho 


\section{Essaouira}

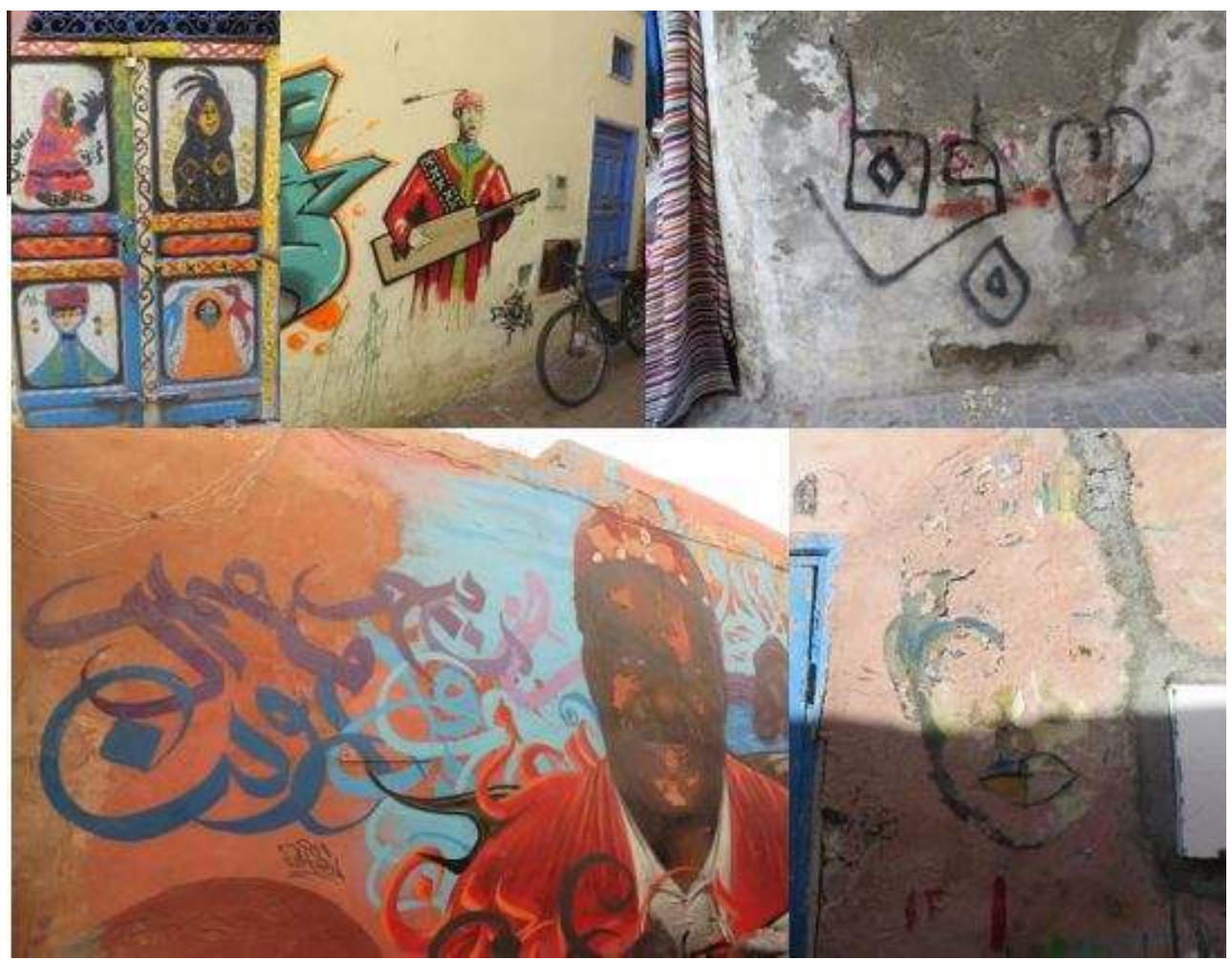

Essaouira 2015.Photos Alain Bertho

\section{Dakar}

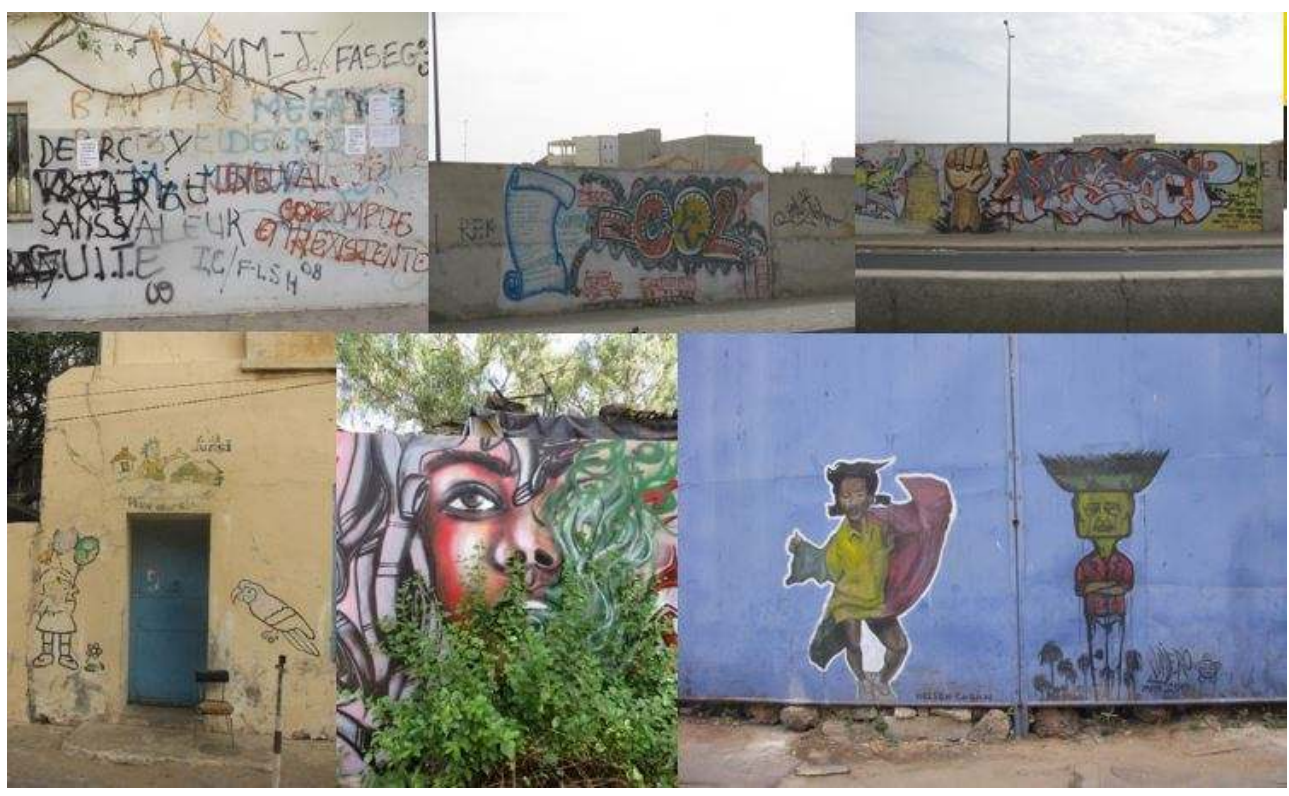

Dakar 2012 Photos Alain Bertho 


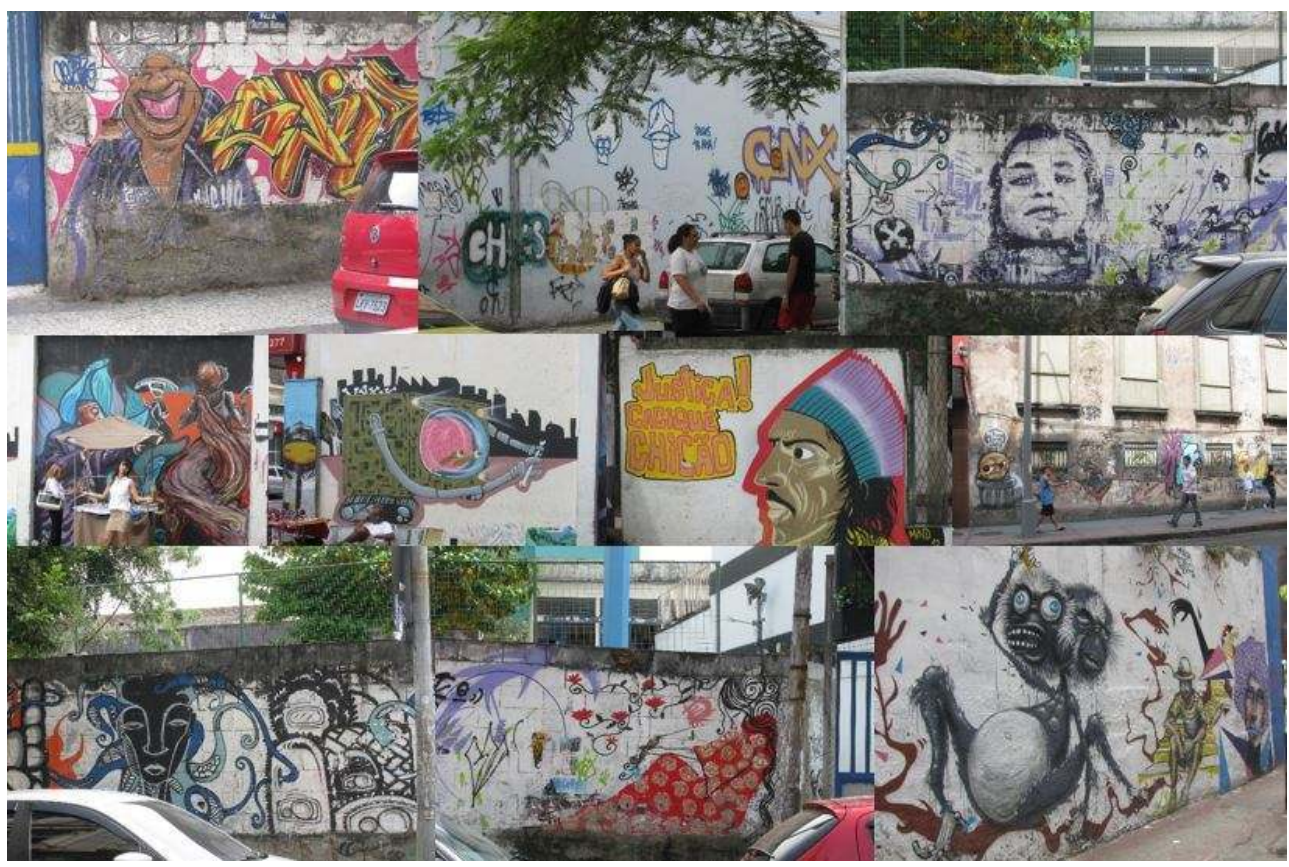

RIO 2013. PHOTOS ALAIN BERTHO

\section{Lire les murs de la ville et en faire le récit}

L'anthropologue Clifford Geertz ${ }^{28}$ nous conviait à lire la société comme un texte pardessus l'épaule de ses acteurs. L'ethnologue qui est pour lui d'abord un auteur, livre le récit d'une vie brièvement partagée avec d'autres, donne à lire des cultures orales et matérielles. C'est un passeur de culture.

C'est pourquoi l'ethnologie exige attention à l'autre, temps d'étonnement et d'analyse, patience dans l'apprentissage de l'altérité. C'est ce même étonnement, cette même attention et patience qu'il lui faut déployer pour lire les images vidéo produites par nos contemporains, notamment dans les situations de mobilisation collective, comme des mobilisations visuelles ${ }^{29}$.

Si l'ethnologue veut aujourd'hui lire les villes, il lui faut lire les murs pour lire les images par-dessus l'épaule des artistes et des passants. Les énoncés picturaux que sont les tags, graffiti et autres interventions graphiques publiques doivent être lus comme des énoncés en situation, localisés et datés. Ces énoncés picturaux ne peuvent être réduits à la sociologie de leurs producteurs ou de leurs éventuels « récepteurs ». Ils ne peuvent être analysés de façon fragmentée en fonction des styles, des ambitions artistiques ou de la reconnaissance institutionnelle ou marchande dont ils bénéficient ou non. Ils forment un ensemble vivant qui déterminera le lieu et le sens des interventions graphiques suivantes. Ils sont une dimension à la fois matérielle et symbolique de la situation dont ils travaillent les dissensus.

41 Si nous poursuivons dans le chemin ouvert par Geertz, il reste la question du récit d'enquête qui se pose au terme de chaque terrain ethnologique. On voit bien que si l'image est devenue un langage vernaculaire omniprésent tant des mobilisations que de la 
ville, il est difficile de faire abstraction de ce langage pour en rendre compte. L'image ne peut pas être réduite à une illustration du propos du chercheur : il doit se l'approprier comme un élément de son propre langage.

\section{APPENDIXES}

\section{Annexes}

Murs du Maroc - juillet 2015

Murs de Naples 2015

Murs de Rio de Janeiro - septembre 2012

Murs de Sicile : Graffs à Catane, Palerme, Enna... - juillet août 2012

Murs de Dakar - 2011-2012

\section{NOTES}

1. Alain Bertho, «L'obscurité du contemporain », Mondes Contemporains, $1^{\circ}$ sem 2012 et « La fin de la politique? », in Ethnographiques.org, « L'anthropologie face aux ruptures », mai 2014

2. Giorgio Agamben, La communauté qui vient, 1990

3. Mitchell, WJT Picture Theory. Essays on Verbal and Visual Representation Chicago : University of Chicago Press, 1994

4. Jacques Rancière, Le partage du sensible. Esthétique et politique ; La Fabrique éditions, 2000

5. Meg McLagan et Yates McKee éd., Sensible politics, Zone book New York, 2012

6. André Gunthert, L'Image partagée. La photographie numérique, Paris, Textuel, 2015

7. Alain Bertho "Soulèvements contemporains et mobilisations visuelles", Socio $n^{\circ} 2$, pages 217-228 et "Énoncés visuels des mobilisations : autoportraits des peuples ", in Anthropologie et sociétés, « Reconnaissances et stratégies médiatiques » 2015

8. Olivier Blondeau, Laurence Allard, dirs., Devenir média. L'activisme sur Internet, entre défection et expérimentation, Paris, Éd. Amsterdam, 2007

9. Alain Bertho, «Le syndrome de Matrix: les mobilisations collectives entre les réseaux numériques et les occupations urbaines ", conférence à la bibliothèque universitaire des langues et civilisations (BULAC), 22 janvier 2013, cycle "La contestation du pouvoir ». Enregistrement disponible sur le site Internet:http://www.bulac.fr/action-culturelle/conferences-les-mardis-de-labulac/reconquerir-lespace-public-les-fondements-de-lengagement/. Matrix ou La Matrice est un film australo-américain de science-fiction, réalisé par Lana et Andy Wachowski en 1999.

10. Focaal, Number 66, Summer 2013. Dossier «Forging the urban Commons », Alain Bertho « Urban commons and urban struggles »

11. http://quandlesmurspalent.blogspot.fr/2012/01/poemes-gribouilles-dans-les-toilettes.html 12. Pedro ARAYA : Des choses écrites - Ecritures exposées en situation de dissensus au Chili, sous la direction de Béatrice Fraenkel, soutenue à l'Ecole des Hautes Etudes en Sciences Sociales le 19 janvier 2015 
13. Francesca Cozzolino, "L'histoire complexe du muralisme en Sardaigne. L'invention d'une tradition de peinture murale et ses multiples influences. ", Nuevo Mundo Mundos Nuevos [En ligne], Images, mémoires et sons, mis en ligne le 30 janvier 2014, consulté le 01 novembre 2015. URL : http://nuevomundo.revues.org/66333 ; DOI : 10.4000/nuevomundo.66333

14. Alain MIOSSEC, Murals d'Irlande Du Nord: Quel avenir après cent années de pratique communautaire?, Rennes, Lannion TIR-CRBC, 2011. Bill Rolston, Drawing Support : Murals in the North of Ireland, Belfast, Beyond the Pale Publications, 1994 ; Drawing Support 2 : Murals of War and Peace, Belfast, Beyond the Pale Publications, 1995

15. Francesca Cozzolino et Ariela Epstein, «Un siècle de peinture murale, Fonctions et dynamiques comparées ", Nuevo Mundo Mundos Nuevos [En ligne], Images, mémoires et sons, mis en ligne le 30 janvier 2014, consulté le 02 novembre 2015. URL: http:// nuevomundo.revues.org/66325

16. Françoise Madray-Lesigne, Jacques Bres, Parole ouvrière autour de Ladrecht, Edition sociales, 1986.

17. Maurice Laurent, Jean Marc Stetka, Bedel, Mansour Bekkada, Alain Gouédard, Michel Trani, Bernard Vaissière, Evelyne Bourré, Gibert St Pré, Roma, Merlin, Astor...

18. Diaporama par Marc Balters http://www.cevennes-photo.fr/fresque/diaporama fresques.html

19. Alain Bertho « Nous n'avons vu que des ombres", in Mouvement, dossier sur les événements de novembre $2005, n^{\circ} 44$ avril mai 2006.

20. Peintre urbain français alias Zoo Project mort en juillet 2013 à l'âge de 23 ans à Détroit (ÉtatsUnis).

21. Athens artist puts flags http://blog.globalstreetart.com/post/25088834194/athens-artist-bputs-flags-of-global-issues-on

La crise grecque inspire les murs http://www.lapresse.ca/photos/voyage/ europe/201206/19/12-7822-la-crise-grecque-inspire-les-murs-dathenes.php

Greek elections and Street art http://athensfever.blogspot.fr/2012/05/greek-elections-andstreet-art.html

Spray pailt and foment : street from Athens http://www.bloomberg.com/slideshow/2012-06-19/ spray-paint-and-foment-street-art-from-athens.html\#slide11

Street art : la crise insp_ire les murs d'Athènes : http://culturebox.francetvinfo.fr/street-art-lacrise-inspire-les-murs-dathenes-102155

22. Des murs pour voir http://www.tunisiartgalleries.com/index.php? option=com_content\&view=article\&id=1137:les-murs-pour-voir-

\&catid=3:tagpressinternational\&Itemid=71

Les armes du street-art tunisien avec ZED https://scribium.com/moniq-akkari/a/les-armes-dustreet-art-tunisien-avec-zed/

Street Art in Tunis - Revolution http://www.artkuwait.org/2011/06/street-art-in-tunisrevolution.html

The Revolutionary Art: Street Art Before and After the Tunisian Revolution http:// digitalcollections.sit.edu/cgi/viewcontent.cgi?article=2130\&context=isp_collection

23. Le «street art» poursuit sa révolution au Caire - LeMonde.fr http://www.lemonde.fr/ afrique/infographe/2011/09/05/le-street-art-poursuit-sa-revolution-au-

caire_1566528_3212.html

Cairo Street Art After the Revolution: Zamalek | suzeeinthecity https:// suzeeinthecity.wordpress.com/2011/06/11/cairo-street-art-after-the-revolution-zamalek/

Themba Lewis Photography > Cairo Street Art http://thembalewis.com/street-art suzeeinthecity | Just another WordPress.com site https://suzeeinthecity.wordpress.com/ The revolution will be painted:Cairo street art| rabble.ca http://rabble.ca/blogs/bloggers/ jesse/2011/06/revolution-will-be-painted-cairo-street-art 
nadara: graffitis post revolution - post revolution street art - al ketaba ... http:// nadara.blogspot.fr/2011/06/graffitis-post-revolution-post.html

The Maturing of Street Art in Cairo - NYTimes.com http://www.nytimes.com/2011/07/28/ world/middleeast/28iht-M28-EGYPT-TAGS.html?pagewanted=all\&_r=0

24. Katia Fersing, Murs blancs, peuples muets entre visibilité et invisibilité, ethnographie des pratiques de graff vandales et semi-légales, 490 pages, Thèse de doctorat, Nice 2011

25. Maria Baredo, Les inscriptions urbaines de Banksy: entre oxymore et paralipse, Xe Congrès de l'Association internationale de sémiotique visuelle, Sep 2012, Buenos Aires, Argentina. JAMES BRASSETT, «British irony, global justice: a pragmatic reading of Chris Brown, Banksy and Ricky Gervais ", Review of International Studies, Review of International Studies / Volume 35 / Issue 01 / January 2009, pp 219-245.

26. Arjun Appadurai, Après le colonialisme les conséquences cultuelles de la globalisation, Payot, 2001. ( Modernity at Large. Cultural Dimensions of Globalization ,1996)

27. On reliera à ce propos le remarquable petit livre de Jean Claude Warnier, la Mondialisation de la culture, La Découverte, 2008 ( $4^{\circ}$ édition)

28. Savoir local, savoir global. Les lieux du savoir, PUF, 1986, 2012 et Ici et là-bas. L'anthropologue comme auteur, Métailié, 1992

29. Ulrike Riboni , "Représentations mobilisatrices et stratégies visuelles pour convaincre et fédérer dans les productions vidéo des soulèvements arabes ", in Bénédicte Rochet, Anne Roekens e. a. (dir.), Quand L'image(dé)mobilise! Iconographies et mouvements sociaux au XXe siècle, Presses universitaires de Namur, 2015.

\section{ABSTRACTS}

The graphic interventions on the walls of cities should be read as statements in situations. Political aesthetics of riots and insurrections of 2011 or localized urban statement of dissent, these pictures contemporaneously articulate singular and common, the local and the global. They fit the "pictorial turn" in the breakdown of social and political groups that marked the end of the 20th century. The investigation into his public image opens a new field for ethnology and raises new writing requirements.

Les interventions graphiques sur les murs des villes doivent être lues comme des énoncés en situations. Esthétiques politiques des émeutes et des insurrections de 2011 ou énoncés de dissensus urbains localisés, ces images articulent de façon contemporaine le singulier et le commun, le local et le mondial. Elles inscrivent le pictural turn dans la rupture des collectifs sociaux et politiques qui a marqué la fin du XXe siècle. L'enquête sur ses images publiques ouvre un nouveau champ pour l'ethnologie et pose de nouvelles exigences d'écriture.

\section{INDEX}

Chronological index: XXème siècle, XXIème siècle Geographical index: Monde 
AUTHOR

ALAIN BERTHO

Professeur d'anthropologie, Université de Paris 8, UMR LAVUE 